\title{
Conformations and Tautomers of Tetracycline
}

Olaf G. Othersen, Frank Beierlein, Harald Lanig and Timothy Clark*

Computer-Chemie-Centrum, Friedrich-Alexander-Universität Erlangen-Nürnberg, Nägelsbachstraße 25, 91052 Erlangen, Germany.

\section{Supporting Information (17 pages)}


Table S1: Calculated total (a.u.) and zero-point energies (ZPE, kcal mol ${ }^{-1}$ ) for neutral tetracycline conformations and tautomers.

\begin{tabular}{|c|c|c|c|c|c|c|}
\hline Species & $\begin{array}{l}\text { Total Energy } \\
\text { (B3LYP) }\end{array}$ & ZPE & $\begin{array}{c}\text { Free Energy } \\
\quad(298 K)\end{array}$ & $\begin{array}{l}\text { Total Energy } \\
\text { (PCM) }\end{array}$ & $\begin{array}{l}\text { Free Energy } \\
(298 \mathrm{~K}, \mathrm{PCM})\end{array}$ & $\begin{array}{c}\text { Total Energy } \\
\text { (MP2//B3LYP) }\end{array}$ \\
\hline \multirow[t]{2}{*}{ N1 } & \multirow[t]{2}{*}{-1564.09568} & \multirow[t]{2}{*}{281.6} & \multirow[t]{2}{*}{-1563.70304} & -1564.11403 & -1563.72139 & \multirow[t]{2}{*}{-1559.46558} \\
\hline & & & & $-1564.12129^{a}$ & $-1563.72865^{\mathrm{a}}$ & \\
\hline $\mathbf{N} 2$ & -1564.08507 & 281.5 & -1563.69319 & -1564.10671 & -1563.71483 & -1559.45830 \\
\hline $\mathbf{N 3}$ & -1564.10012 & 281.5 & -1563.70783 & -1564.10928 & -1563.71699 & -1559.46849 \\
\hline $\mathbf{N 4}$ & -1564.09327 & 281.0 & -1563.70253 & -1564.10707 & -1563.71633 & -1559.45937 \\
\hline N5 & -1564.07453 & 281.0 & -1563.68408 & -1564.09725 & -1563.70680 & -1559.44044 \\
\hline N6 & -1564.07990 & 281.3 & -1563.68812 & -1564.09457 & -1563.70279 & -1559.44922 \\
\hline Z1 & -1564.06307 & 282.8 & -1563.66888 & -1564.10295 & -1563.70876 & -1559.44139 \\
\hline $\mathbf{Z 2}$ & -1564.04723 & 281.9 & -1563.65480 & $-1564.10369^{\mathrm{a}}$ & $-1563.71125^{\mathrm{a}}$ & -1559.42833 \\
\hline $\mathbf{Z 3}$ & -1564.05191 & 281.0 & -1563.66014 & -1564.09982 & -1563.70805 & -1559.42825 \\
\hline $\mathbf{Z 4}$ & -1564.05708 & 281.5 & -1563.665864 & -1564.10145 & -1563.71023 & -1559.43113 \\
\hline $\mathbf{Z 5}$ & -1564.05361 & 282.5 & -1563.66048 & -1564.09641 & -1563.70328 & -1559.43392 \\
\hline Z6 & -1564.05521 & 281.5 & -1563.66249 & -1564.10111 & -1563.70839 & -1559.42990 \\
\hline $\mathbf{Z 7}$ & -1564.06269 & 283.2 & -1563.66726 & -1564.10136 & -1563.70593 & -1559.43884 \\
\hline $\mathbf{Z 8}$ & -1564.05570 & 281.4 & -1563.66329 & -1564.10076 & -1563.70835 & -1559.42914 \\
\hline Z9 & -1564.06823 & 281.9 & -1563.67501 & -1564.1019 & -1563.70869 & -1559.44116 \\
\hline Z10 & -1564.03709 & 281.3 & -1563.64561 & -1564.09806 & -1563.70658 & -1559.41186 \\
\hline Z11 & -1564.04380 & 282.2 & -1563.65077 & -1564.10142 & -1563.70839 & -1559.41683 \\
\hline Z12 & -1564.05921 & 282.4 & -1563.66573 & -1564.09529 & -1563.70181 & -1559.43848 \\
\hline Z13 & -1564.04905 & 281.7 & -1563.65593 & -1564.09707 & -1563.70395 & -1559.42500 \\
\hline Z14 & -1564.06308 & 281.4 & -1563.63076 & -1564.09691 & -1563.70459 & -1559.43768 \\
\hline Z15 & -1564.05642 & 282.5 & -1563.66202 & -1564.09409 & -1563.69969 & -1559.43459 \\
\hline Z16 & -1564.04962 & 281.7 & -1563.65656 & -1564.09510 & -1563.70204 & -1559.42503 \\
\hline Z17 & -1564.05398 & 283.3 & -1563.65870 & -1564.09392 & -1563.69864 & -1559.43184 \\
\hline Z18 & -1564.04171 & 282.2 & -1563.64746 & -1564.09392 & -1563.69967 & -1559.41725 \\
\hline Z19 & -1564.04282 & 281.4 & -1563.65052 & -1564.09271 & -1563.70040 & -1559.41665 \\
\hline $\mathbf{Z 2 0}$ & -1564.04252 & 281.7 & -1563.652207 & -1564.08942 & -1563.69911 & -1559.41616 \\
\hline
\end{tabular}

${ }^{a}$ The UAHF-PCM calculation failed consistently for structure $\mathbf{Z 2}$. A PCM-calculation using the default isodensity surface (IEPCM) was therefore compared with an analogous calculation for the most stable species, N1. 
Table S2: Hydrogen bonding distances. The column headings give the hydrogen-bond donor and the acceptor (oxygen or nitrogen) is given in parentheses before the $\mathrm{H}$-acceptor distance in Ångstrøm.

\begin{tabular}{|c|c|c|c|c|c|c|c|c|}
\hline Species & $O 10(11)$ & $012 a$ & 01 & $\mathbf{O 3}$ & 011 & 012 & amine & Amide $O$ \\
\hline N1 & 1.668 & (N) 1.847 & & (A) 1.492 & & (11) 1.615 & & \\
\hline $\mathbf{N} 2$ & 1.626 & (N) 1.809 & & (A) 1.488 & & (12a) 1.918 & & \\
\hline N3 & 1.668 & (1) 1.956 & & (A) 1.509 & & (11) 1.602 & & \\
\hline N4 & 1.681 & (12) 2.073 & & (A) 1.514 & & (11) 1.604 & & \\
\hline N5 & 1.633 & (12) 2.153 & & (A) 1.488 & & (1) 1.750 & & \\
\hline N6 & 1.628 & (1) 2.111 & & (A) 1.467 & & (1) 1.856 & & \\
\hline $2 \mathbf{Z 1}$ & 1.675 & (12) 2.250 & & & & (11) 1.600 & (12a) 1.852 & \\
\hline $\mathbf{Z 2}$ & 1.606 & (12) 1.970 & (12) 1.554 & & & & (12a) 1.832 & \\
\hline $\mathbf{Z 3}$ & 1.586 & (12) 1.553 & & (A) 1.476 & & & (12a) 1.553 & \\
\hline $\mathrm{Z4}$ & 1.667 & (12) 2.242 & & & & (11) 1.615 & (3) 1.622 & \\
\hline $\mathbf{Z 5}$ & 1.627 & \begin{tabular}{|c|} 
(1) 2.495 \\
(12) 2.419
\end{tabular} & & & & (1) 1.641 & (12a) 1.919 & \\
\hline Z6 & 1.583 & (12) 1.563 & & & & & (12a) 1.635 & (3) 1.572 \\
\hline $\mathbf{Z 7}$ & 1.649 & (1) 1.833 & & & & (11) 1.613 & (6) 1.897 & \\
\hline $\mathbf{Z 8}$ & 1.588 & (12) 1.565 & (A) 1.500 & & & & (12a) 1.661 & \\
\hline Z9 & 1.661 & (1) 1.849 & & & & (11) 1.611 & (3) 1.499 & \\
\hline Z10 & 1.576 & (12) 1.645 & & (A) 1.453 & & & (3) 2.193 & \\
\hline $\mathbf{Z 1 1}$ & 1.574 & (12) 1.673 & & & & & (3) 1.976 & (3) 1.639 \\
\hline Z12 & 1.760 & (12) 2.102 & & & (12) 1.542 & & (12a) 1.831 & \\
\hline $\mathrm{Z13}$ & 1.505 & (1) 2.104 & & & & & (6) 1.722 & (3) 1.587 \\
\hline Z14 & 1.748 & (1) 1.854 & & & (12) 1.517 & & (3) 1.485 & \\
\hline Z15 & 1.740 & (1) 1.838 & & & (12) 1.490 & & (6) 1.911 & \\
\hline Z16 & 1.532 & (1) 2.390 & (A) 1.503 & & & & (6) 1.747 & \\
\hline Z17 & 1.599 & (1) 2.026 & & & & (1) 1.735 & (6) 1.809 & \\
\hline Z18 & 1.558 & (1) 2.109 & & & & & (3) 1.776 & (3) 1.636 \\
\hline Z19 & 1.565 & (1) 2.131 & (A) 1.503 & & & & (3) 1.747 & \\
\hline $\mathbf{Z 2 0}$ & 1.618 & (12) 2.206 & & & & (1) 1.670 & (3) 1.744 & \\
\hline
\end{tabular}


Table S3: Root-mean-square deviations (RMSD, $\AA$ ) between the non-hydrogen-atom skeletons of the minimum-energy structures found for neutral tetracycline. All RMSD-values less than $1.0 \AA$ are shown in bold.

\begin{tabular}{|c|c|c|c|c|c|c|c|c|c|c|c|c|c|c|c|c|c|c|c|c|c|c|c|c|c|c|}
\hline & N1 & N2 & N3 & N4 & N5 & N6 & & & & & & & & & & & & & & & & & & & & \\
\hline N1 & 0 & & & & & & & & & & & & & & & & & & & & & & & & & \\
\hline N2 & $\mathbf{0 , 3}$ & 0 & & & & & & & & & & & & & & & & & & & & & & & & \\
\hline N3 & 1,8 & 1,9 & 0 & & & & & & & & & & & & & & & & & & & & & & & \\
\hline N4 & 0,4 & 0,6 & 1,8 & 0 & & & & & & & & & & & & & & & & & & & & & & \\
\hline N5 & $\mathbf{0 , 4}$ & $\mathbf{0 , 6}$ & 1,7 & $\mathbf{0 , 3}$ & 0 & & & & & & & & & & & & & & & & & & & & & \\
\hline N6 & 1,6 & 1,7 & 0,5 & 1,5 & 1,4 & 0 & Z1 & $\mathbf{Z 2}$ & $\mathbf{Z 3}$ & $\mathbf{Z 4}$ & $\mathrm{Z5}$ & $\mathbf{Z 6}$ & $\mathbf{Z 7}$ & $\mathbf{Z 8}$ & Z9 & Z10 & Z11 & Z12 & $\mathrm{Z13}$ & Z14 & $\mathrm{Z15}$ & Z16 & $\mathrm{Z17}$ & Z18 & Z19 & $\mathbf{Z 2 0}$ \\
\hline $\mathrm{Z1}$ & $\mathbf{0 , 7}$ & $\mathbf{0 , 8}$ & 1,7 & $\mathbf{0 , 7}$ & $\mathbf{0 , 6}$ & 1,5 & 0 & & & & & & & & & & & & & & & & & & & \\
\hline $\mathbf{Z 2}$ & $\mathbf{0 , 7}$ & $\mathbf{0 , 8}$ & 1,7 & $\mathbf{0 , 7}$ & $\mathbf{0 , 6}$ & 1,5 & 0,1 & 0 & & & & & & & & & & & & & & & & & & \\
\hline $\mathbf{Z 3}$ & $\mathbf{0 , 7}$ & $\mathbf{0 , 6}$ & 1,9 & $\mathbf{0 , 7}$ & $\mathbf{0 , 8}$ & 1,7 & $\mathbf{0 , 5}$ & $\mathbf{0 , 5}$ & 0 & & & & & & & & & & & & & & & & & \\
\hline $\mathbf{Z 4}$ & $\mathbf{0 , 9}$ & 1,1 & 1,7 & $\mathbf{0 , 7}$ & $\mathbf{0 , 8}$ & 1,5 & 1,0 & 1,0 & 1,1 & 0 & & & & & & & & & & & & & & & & \\
\hline Z5 & $\mathbf{0 , 7}$ & $\mathbf{0 , 8}$ & 1,6 & $\mathbf{0 , 7}$ & $\mathbf{0 , 6}$ & 1,4 & 0,2 & $\mathbf{0 , 1}$ & $\mathbf{0 , 6}$ & $\mathbf{1 , 0}$ & 0 & & & & & & & & & & & & & & & \\
\hline $\mathrm{Z6}$ & 0,7 & 0,6 & 1,9 & $\mathbf{0 , 8}$ & $\mathbf{0 , 8}$ & 1,7 & 0,5 & 0,6 & $\mathbf{0 , 1}$ & 1,1 & 0,6 & 0 & & & & & & & & & & & & & & \\
\hline $\mathbf{Z 7}$ & 2,0 & 2,1 & $\mathbf{0 , 5}$ & 1,9 & 1,9 & 0,6 & 1,9 & 1,9 & 2,2 & 1,8 & 1,9 & 2,2 & 0 & & & & & & & & & & & & & \\
\hline Z8 & $\mathbf{0 , 8}$ & $\mathbf{0 , 8}$ & 1,9 & $\mathbf{0 , 9}$ & $\mathbf{0 , 9}$ & 1,7 & $\mathbf{0 , 7}$ & $\mathbf{0 , 7}$ & $\mathbf{0 , 6}$ & $\mathbf{1 , 0}$ & $\mathbf{0 , 8}$ & $\mathbf{0 , 6}$ & 2,2 & 0 & & & & & & & & & & & & \\
\hline $\mathbf{Z 9}$ & 2,0 & 2,2 & $\mathbf{0 , 8}$ & 1,9 & 1,9 & 1,0 & 1,9 & 1,9 & 2,2 & 1,8 & 1,9 & 2,2 & $\mathbf{0 , 7}$ & 2,2 & 0 & & & & & & & & & & & \\
\hline Z10 & $\mathbf{0 , 3}$ & $\mathbf{0 , 4}$ & 1,9 & $\mathbf{0 , 2}$ & $\mathbf{0 , 4}$ & 1,6 & $\mathbf{0 , 7}$ & $\mathbf{0 , 7}$ & $\mathbf{0 , 7}$ & $\mathbf{0 , 8}$ & $\mathbf{0 , 8}$ & $\mathbf{0 , 7}$ & 2,0 & $\mathbf{0 , 9}$ & 2,1 & 0 & & & & & & & & & & \\
\hline Z11 & $\mathbf{0 , 4}$ & $\mathbf{0 , 5}$ & 1,9 & $\mathbf{0 , 2}$ & $\mathbf{0 , 4}$ & 1,6 & $\mathbf{0 , 7}$ & $\mathbf{0 , 7}$ & $\mathbf{0 , 7}$ & $\mathbf{0 , 8}$ & 0,8 & $\mathbf{0 , 7}$ & 2,0 & $\mathbf{0 , 9}$ & 2,0 & 0,1 & 0 & & & & & & & & & \\
\hline $\mathrm{Z12}$ & $\mathbf{0 , 7}$ & $\mathbf{0 , 8}$ & 1,7 & $\mathbf{0 , 7}$ & $\mathbf{0 , 7}$ & 1,5 & $\mathbf{0 , 1}$ & 0,2 & $\mathbf{0 , 5}$ & 1,0 & $\mathbf{0 , 3}$ & $\mathbf{0 , 5}$ & 1,9 & $\mathbf{0 , 7}$ & 1,9 & $\mathbf{0 , 7}$ & $\mathbf{0 , 7}$ & 0 & & & & & & & & \\
\hline Z13 & 2,2 & 2,3 & $\mathbf{0 , 6}$ & 2,1 & 2,0 & 0,8 & 2,1 & 2,1 & 2,3 & 2,0 & 2,0 & 2,3 & $\mathbf{0 , 3}$ & 2,3 & $\mathbf{0 , 8}$ & 2,2 & 2,2 & 2,1 & 0 & & & & & & & \\
\hline $\mathrm{Z14}$ & 2,1 & 2,2 & 0,8 & 1,9 & 1,9 & 1,0 & 1,9 & 1,9 & 2,2 & 1,8 & 1,9 & 2,3 & $\mathbf{0 , 7}$ & 2,2 & 0,1 & 2,1 & 2,1 & 1,9 & $\mathbf{0 , 8}$ & 0 & & & & & & \\
\hline Z15 & 2,0 & 2,2 & 0,5 & 2,0 & 1,9 & 0,7 & 2,0 & 2,0 & 2,2 & 1,9 & 1,9 & 2,2 & $\mathbf{0 , 1}$ & 2,2 & 0,7 & 2,1 & 2,1 & 2,0 & $\mathbf{0 , 3}$ & 0,7 & 0 & & & & & \\
\hline Z16 & 2,1 & 2,3 & $\mathbf{0 , 8}$ & 2,1 & 2,0 & 0,9 & 2,0 & 2,0 & 2,3 & 1,9 & 2,0 & 2,3 & 0,6 & 2,2 & 0,9 & 2,2 & 2,2 & 2,0 & 0,6 & 0,9 & 0,6 & 0 & & & & \\
\hline Z17 & 1,8 & 2,0 & $\mathbf{0 , 5}$ & 1,8 & 1,7 & $\mathbf{0 , 5}$ & 1,8 & 1,8 & 2,0 & 1,7 & 1,8 & 2,0 & $\mathbf{0 , 3}$ & 2,0 & $\mathbf{0 , 8}$ & 1,9 & 1,9 & 1,8 & $\mathbf{0 , 5}$ & $\mathbf{0 , 9}$ & $\mathbf{0 , 4}$ & $\mathbf{0 , 7}$ & 0 & & & \\
\hline Z18 & 2,1 & 2,3 & 0,9 & 2,0 & 2,0 & 1,1 & 2,0 & 2,0 & 2,3 & 1,9 & 2,0 & 2,3 & $\mathbf{0 , 8}$ & 2,3 & 0,2 & 2,2 & 2,2 & 2,0 & $\mathbf{0 , 8}$ & 0,2 & $\mathbf{0 , 8}$ & 0,9 & $\mathbf{0 , 9}$ & 0 & & \\
\hline Z19 & 2,2 & 2,3 & 1,0 & 2,1 & 2,0 & 1,2 & 2,0 & 2,0 & 2,4 & 1,8 & 2,0 & 2,4 & $\mathbf{1 , 0}$ & 2,3 & $\mathbf{0 , 6}$ & 2,2 & 2,2 & 2,0 & 1,0 & $\mathbf{0 , 6}$ & $\mathbf{0 , 9}$ & $\mathbf{0 , 8}$ & 1,1 & $\mathbf{0 , 6}$ & 0 & \\
\hline $\mathrm{Z20}$ & $\mathbf{0 , 9}$ & 1,0 & 1,7 & $\mathbf{0 , 7}$ & $\mathbf{0 , 7}$ & 1,5 & $\mathbf{0 , 9}$ & $\mathbf{0 , 9}$ & 1,1 & $\mathbf{0 , 2}$ & 0,9 & 1,1 & 1,8 & $\mathbf{0 , 9}$ & 1,8 & $\mathbf{0 , 8}$ & $\mathbf{0 , 7}$ & $\mathbf{0 , 9}$ & 2,0 & 1,8 & 1,9 & 1,9 & 1,7 & 1,9 & 1,8 & 0 \\
\hline
\end{tabular}


Table S4: Calculated infrared frequencies $\left(\mathrm{cm}^{-1}\right.$ in ascending order) for the 26 tetracycline structures.

\begin{tabular}{|c|c|c|c|c|c|c|c|c|c|c|c|c|}
\hline N1 & $\mathbf{N 2}$ & N3 & N4 & N5 & N6 & Z1 & $\mathbf{Z 2}$ & $\mathbf{Z 3}$ & Z4 & $\mathbf{Z 5}$ & Z6 & Z7 \\
\hline 22.98 & 20.05 & 21.12 & 19.19 & 20.40 & 18.71 & 25.37 & 24.57 & 21.12 & 21.61 & 23.45 & 19.28 & 28.09 \\
\hline 35.16 & 32.37 & 29.11 & 29.34 & 26.25 & 28.89 & 36.38 & 32.56 & 34.00 & 30.90 & 34.50 & 33.07 & 33.23 \\
\hline 40.32 & 41.13 & 48.46 & 36.69 & 35.06 & 47.43 & 48.13 & 44.66 & 47.70 & 41.71 & 47.53 & 47.35 & 45.01 \\
\hline 59.55 & 49.62 & 57.91 & 59.27 & 49.88 & 57.48 & 55.18 & 50.44 & 60.65 & 58.28 & 54.14 & 58.22 & 59.14 \\
\hline 69.37 & 70.21 & 59.90 & 69.25 & 67.41 & 70.55 & 70.29 & 76.19 & 74.33 & 64.78 & 69.30 & 73.99 & 75.04 \\
\hline 79.59 & 79.70 & 88.53 & 84.70 & 79.04 & 77.72 & 85.27 & 83.61 & 80.45 & 71.89 & 83.69 & 80.38 & 80.96 \\
\hline 101.54 & 94.64 & 107.56 & 94.55 & 96.45 & 100.42 & 94.72 & 87.89 & 97.82 & 94.24 & 90.52 & 98.29 & 104.02 \\
\hline 105.18 & 103.46 & 118.20 & 103.97 & 102.82 & 106.50 & 103.50 & 100.56 & 107.23 & 103.00 & 100.02 & 108.10 & 120.87 \\
\hline 115.23 & 111.38 & 121.60 & 114.34 & 110.29 & 129.93 & 105.15 & 103.10 & 116.69 & 105.21 & 103.42 & 136.37 & 132.13 \\
\hline 136.84 & 129.70 & 134.95 & 135.75 & 133.88 & 150.29 & 133.79 & 131.24 & 137.47 & 130.63 & 127.28 & 137.49 & 145.47 \\
\hline 166.78 & 155.12 & 166.04 & 145.49 & 147.52 & 154.22 & 153.70 & 147.57 & 154.28 & 150.03 & 147.24 & 154.96 & 164.37 \\
\hline 173.79 & 165.53 & 172.75 & 160.31 & 157.72 & 172.26 & 165.97 & 156.56 & 164.00 & 159.96 & 157.14 & 167.33 & 176.19 \\
\hline 177.31 & 174.24 & 177.26 & 176.15 & 177.25 & 179.80 & 180.61 & 173.85 & 174.47 & 175.17 & 170.64 & 174.19 & 180.88 \\
\hline 201.25 & 193.30 & 189.28 & 195.40 & 207.29 & 206.97 & 188.64 & 182.35 & 195.45 & 187.35 & 182.67 & 195.06 & 197.73 \\
\hline 212.89 & 209.69 & 207.45 & 207.62 & 212.36 & 210.01 & 200.38 & 195.63 & 210.43 & 199.61 & 190.25 & 210.65 & 199.38 \\
\hline 229.69 & 226.41 & 219.98 & 224.18 & 223.60 & 211.42 & 202.38 & 198.55 & 216.74 & 209.77 & 198.90 & 223.62 & 219.96 \\
\hline 233.40 & 232.10 & 233.08 & 228.33 & 227.80 & 222.02 & 217.07 & 216.89 & 236.18 & 217.90 & 209.29 & 236.00 & 222.51 \\
\hline 238.27 & 236.76 & 238.64 & 235.35 & 232.24 & 238.69 & 225.79 & 231.76 & 242.72 & 226.66 & 223.81 & 240.26 & 229.27 \\
\hline 241.72 & 246.17 & 242.30 & 237.51 & 244.66 & 243.18 & 232.54 & 236.88 & 248.95 & 232.21 & 230.23 & 251.66 & 234.13 \\
\hline 267.22 & 267.06 & 252.97 & 244.75 & 249.04 & 245.72 & 251.28 & 244.31 & 254.94 & 240.13 & 245.79 & 257.37 & 241.16 \\
\hline 272.20 & 270.76 & 258.10 & 259.39 & 264.69 & 266.77 & 262.91 & 268.38 & 276.19 & 246.21 & 262.58 & 277.39 & 247.76 \\
\hline 290.94 & 289.15 & 264.13 & 266.25 & 275.44 & 277.80 & 284.48 & 273.39 & 285.04 & 266.89 & 280.55 & 281.08 & 261.43 \\
\hline 301.62 & 300.96 & 279.50 & 285.27 & 289.37 & 292.60 & 288.02 & 289.59 & 300.65 & 279.16 & 284.78 & 293.40 & 279.24 \\
\hline 309.83 & 307.77 & 297.96 & 303.92 & 293.47 & 300.84 & 306.20 & 299.43 & 308.44 & 286.89 & 300.26 & 307.12 & 283.50 \\
\hline 322.08 & 318.44 & 311.15 & 318.39 & 312.79 & 315.30 & 309.77 & 310.87 & 317.25 & 292.85 & 309.14 & 312.17 & 307.33 \\
\hline 329.05 & 326.64 & 321.28 & 324.34 & 317.48 & 324.89 & 322.84 & 316.72 & 323.49 & 312.10 & 312.08 & 322.88 & 316.25 \\
\hline 331.66 & 340.09 & 329.61 & 328.28 & 326.89 & 336.37 & 331.13 & 328.72 & 333.53 & 320.21 & 324.41 & 336.43 & 320.68 \\
\hline 350.25 & 355.17 & 337.22 & 332.26 & 332.58 & 341.33 & 344.89 & 344.45 & 354.81 & 323.02 & 333.56 & 359.35 & 334.70 \\
\hline 360.48 & 362.29 & 356.88 & 352.56 & 341.41 & 358.21 & 350.42 & 351.88 & 363.53 & 335.71 & 348.46 & 370.90 & 344.76 \\
\hline
\end{tabular}




\begin{tabular}{|c|c|c|c|c|c|c|c|c|c|c|c|c|}
\hline 366.08 & 369.23 & 368.34 & 357.46 & 348.24 & 374.00 & 359.26 & 363.96 & 371.22 & 349.68 & 350.77 & 375.61 & 358.43 \\
\hline 370.48 & 374.43 & 377.91 & 369.67 & 370.81 & 379.85 & 365.54 & 375.36 & 375.53 & 363.74 & 373.37 & 383.25 & 367.34 \\
\hline 381.89 & 389.29 & 378.78 & 380.96 & 376.29 & 380.46 & 371.12 & 383.17 & 386.99 & 373.07 & 378.10 & 398.83 & 381.05 \\
\hline 388.66 & 392.93 & 384.62 & 388.70 & 387.12 & 391.35 & 381.71 & 388.24 & 412.89 & 380.18 & 385.11 & 424.02 & 387.97 \\
\hline 392.15 & 405.75 & 396.47 & 396.77 & 394.13 & 403.20 & 393.19 & 416.52 & 418.03 & 397.85 & 396.27 & 427.39 & 405.21 \\
\hline 410.30 & 421.71 & 423.15 & 402.74 & 406.35 & 413.67 & 432.86 & 432.78 & 428.03 & 407.80 & 429.16 & 428.17 & 431.13 \\
\hline 422.03 & 424.87 & 427.34 & 415.56 & 415.62 & 426.52 & 442.17 & 441.50 & 452.15 & 418.36 & 434.70 & 453.43 & 448.93 \\
\hline 454.79 & 439.14 & 430.58 & 426.85 & 435.13 & 430.23 & 457.35 & 450.54 & 457.12 & 445.92 & 439.04 & 458.24 & 452.86 \\
\hline 459.18 & 459.98 & 445.83 & 435.18 & 439.69 & 434.74 & 460.91 & 455.99 & 468.26 & 449.31 & 458.40 & 475.20 & 456.35 \\
\hline 468.87 & 466.10 & 452.89 & 455.51 & 445.66 & 450.29 & 481.19 & 464.49 & 479.90 & 473.28 & 464.81 & 484.46 & 478.23 \\
\hline 486.07 & 476.03 & 475.24 & 462.65 & 466.28 & 458.75 & 482.83 & 469.68 & 488.88 & 492.05 & 470.75 & 492.93 & 481.82 \\
\hline 490.35 & 489.45 & 483.36 & 485.97 & 470.53 & 460.23 & 489.30 & 478.21 & 493.87 & 495.19 & 491.80 & 494.65 & 492.13 \\
\hline 497.72 & 491.29 & 494.10 & 489.34 & 491.40 & 479.21 & 497.58 & 493.27 & 496.37 & 498.09 & 492.02 & 502.65 & 495.57 \\
\hline 502.62 & 500.23 & 499.50 & 495.86 & 494.88 & 492.96 & 502.76 & 498.27 & 504.82 & 505.45 & 501.17 & 511.11 & 509.73 \\
\hline 524.96 & 525.03 & 511.14 & 504.34 & 504.91 & 495.84 & 520.81 & 521.07 & 525.95 & 507.84 & 519.93 & 526.32 & 523.22 \\
\hline 538.43 & 531.17 & 516.72 & 525.05 & 521.57 & 511.88 & 534.94 & 538.04 & 539.59 & 518.77 & 534.11 & 540.25 & 533.94 \\
\hline 545.00 & 542.50 & 529.88 & 529.58 & 535.41 & 521.10 & 539.73 & 542.02 & 548.62 & 529.92 & 542.65 & 547.28 & 549.46 \\
\hline 571.69 & 563.24 & 549.63 & 541.39 & 539.18 & 535.67 & 557.26 & 565.33 & 571.87 & 544.07 & 562.21 & 554.23 & 580.27 \\
\hline 583.84 & 580.68 & 574.86 & 573.66 & 585.38 & 580.69 & 567.91 & 573.21 & 581.20 & 558.47 & 570.07 & 581.29 & 587.37 \\
\hline 604.77 & 584.18 & 586.80 & 584.10 & 596.50 & 586.88 & 586.32 & 588.82 & 600.80 & 565.91 & 593.42 & 600.79 & 603.34 \\
\hline 625.26 & 602.56 & 613.15 & 604.38 & 609.93 & 610.34 & 600.74 & 601.24 & 641.79 & 603.37 & 601.42 & 637.43 & 635.56 \\
\hline 644.29 & 634.94 & 624.11 & 616.30 & 619.62 & 638.89 & 624.61 & 627.69 & 644.69 & 605.84 & 608.56 & 642.77 & 643.98 \\
\hline 652.85 & 645.10 & 644.79 & 645.55 & 645.72 & 644.55 & 644.57 & 642.50 & 656.33 & 632.73 & 623.57 & 658.65 & 652.31 \\
\hline 681.87 & 654.40 & 650.84 & 658.01 & 654.37 & 653.23 & 651.66 & 649.04 & 681.84 & 645.34 & 645.05 & 684.10 & 664.49 \\
\hline 701.13 & 684.69 & 673.53 & 680.78 & 676.42 & 659.37 & 653.41 & 662.56 & 707.20 & 652.25 & 650.58 & 710.80 & 675.87 \\
\hline 709.26 & 695.14 & 699.90 & 701.79 & 692.22 & 683.80 & 691.17 & 684.83 & 713.08 & 672.03 & 683.80 & 713.88 & 679.91 \\
\hline 714.62 & 710.58 & 706.82 & 711.46 & 705.13 & 692.54 & 708.16 & 706.67 & 721.89 & 698.57 & 700.16 & 724.56 & 700.84 \\
\hline 731.39 & 714.16 & 711.46 & 718.02 & 717.54 & 698.27 & 712.63 & 710.15 & 731.24 & 711.85 & 703.11 & 741.78 & 705.36 \\
\hline 739.01 & 738.39 & 739.04 & 721.85 & 725.79 & 710.27 & 715.78 & 712.09 & 743.65 & 722.20 & 717.44 & 747.36 & 736.18 \\
\hline 753.34 & 745.53 & 761.91 & 751.88 & 748.82 & 737.22 & 743.53 & 740.17 & 758.23 & 747.84 & 741.62 & 762.00 & 758.62 \\
\hline 765.80 & 762.44 & 767.60 & 770.28 & 765.97 & 767.71 & 752.73 & 752.69 & 781.38 & 749.59 & 752.48 & 791.27 & 766.96 \\
\hline 777.50 & 769.09 & 780.28 & 780.08 & 782.41 & 768.75 & 769.15 & 769.97 & 793.40 & 780.64 & 772.59 & 795.49 & 780.32 \\
\hline
\end{tabular}




\begin{tabular}{|c|c|c|c|c|c|c|c|c|c|c|c|c|}
\hline 789.38 & 779.69 & 790.68 & 793.50 & 784.54 & 778.58 & 783.45 & 792.21 & 798.73 & 791.32 & 790.19 & 800.44 & 794.95 \\
\hline 798.79 & 796.18 & 798.86 & 800.60 & 797.16 & 798.07 & 796.44 & 795.37 & 810.00 & 796.46 & 799.72 & 816.29 & 800.48 \\
\hline 807.78 & 800.85 & 808.09 & 807.58 & 803.25 & 801.85 & 804.66 & 805.23 & 823.19 & 800.98 & 808.24 & 823.37 & 812.01 \\
\hline 814.82 & 809.92 & 812.83 & 812.54 & 815.24 & 808.41 & 811.84 & 825.93 & 849.03 & 815.60 & 822.81 & 863.66 & 819.70 \\
\hline 823.96 & 826.21 & 831.19 & 824.43 & 821.73 & 832.94 & 823.78 & 850.83 & 865.83 & 818.98 & 856.82 & 866.94 & 825.37 \\
\hline 864.03 & 858.50 & 850.44 & 858.15 & 856.77 & 850.97 & 858.89 & 866.43 & 870.16 & 850.69 & 864.96 & 877.93 & 836.00 \\
\hline 867.97 & 863.19 & 881.58 & 866.88 & 858.80 & 863.18 & 869.51 & 878.89 & 883.70 & 856.20 & 867.06 & 883.21 & 868.37 \\
\hline 881.22 & 866.95 & 896.39 & 881.13 & 861.82 & 864.84 & 880.17 & 885.43 & 912.79 & 875.54 & 876.97 & 915.25 & 881.25 \\
\hline 896.83 & 880.20 & 902.10 & 893.16 & 879.37 & 893.19 & 897.39 & 894.55 & 922.64 & 881.93 & 894.84 & 925.80 & 888.09 \\
\hline 901.23 & 892.64 & 912.64 & 899.61 & 895.16 & 900.06 & 898.81 & 912.96 & 947.54 & 894.37 & 909.47 & 947.68 & 900.21 \\
\hline 949.88 & 949.50 & 916.37 & 947.08 & 944.66 & 906.34 & 911.50 & 947.26 & 957.69 & 937.73 & 912.24 & 953.60 & 920.33 \\
\hline 958.53 & 954.61 & 955.83 & 958.26 & 954.96 & 957.60 & 942.77 & 956.97 & 968.11 & 949.05 & 946.76 & 963.41 & 930.90 \\
\hline 973.39 & 972.38 & 964.86 & 971.09 & 971.70 & 960.74 & 959.83 & 970.77 & 968.94 & 971.75 & 954.35 & 964.91 & 943.82 \\
\hline 984.42 & 980.29 & 974.29 & 984.00 & 979.10 & 973.18 & 968.44 & 975.35 & 975.26 & 974.32 & 965.23 & 967.92 & 969.97 \\
\hline 986.68 & 985.52 & 986.70 & 987.31 & 984.31 & 982.36 & 973.63 & 983.96 & 993.97 & 978.20 & 979.50 & 974.30 & 982.10 \\
\hline 1013.78 & 1004.45 & 1005.68 & 1016.74 & 1003.26 & 1000.37 & 986.36 & 994.62 & 1006.34 & 981.36 & 982.45 & 998.75 & 986.32 \\
\hline 1020.65 & 1016.63 & 1034.16 & 1036.66 & 1049.00 & 1022.94 & 998.45 & 1018.03 & 1027.24 & 1021.86 & 992.08 & 1008.63 & 1000.91 \\
\hline 1053.66 & 1044.84 & 1042.73 & 1055.86 & 1055.92 & 1047.97 & 1020.88 & 1037.10 & 1042.75 & 1041.22 & 1018.37 & 1028.10 & 1003.44 \\
\hline 1054.39 & 1057.62 & 1056.61 & 1059.42 & 1059.70 & 1065.20 & 1039.93 & 1043.97 & 1064.30 & 1049.09 & 1033.84 & 1044.08 & 1027.99 \\
\hline 1066.54 & 1063.11 & 1069.78 & 1070.76 & 1065.50 & 1077.66 & 1043.08 & 1058.28 & 1066.68 & 1061.69 & 1040.57 & 1064.75 & 1056.06 \\
\hline 1079.23 & 1075.85 & 1085.12 & 1072.65 & 1075.34 & 1079.09 & 1063.26 & 1064.07 & 1079.55 & 1083.62 & 1062.69 & 1078.63 & 1063.73 \\
\hline 1084.43 & 1082.90 & 1087.67 & 1086.05 & 1085.46 & 1088.01 & 1074.14 & 1075.42 & 1084.67 & 1087.16 & 1075.50 & 1085.25 & 1079.27 \\
\hline 1090.19 & 1087.11 & 1109.16 & 1089.81 & 1087.45 & 1097.95 & 1085.36 & 1084.73 & 1089.40 & 1088.87 & 1084.30 & 1090.00 & 1100.21 \\
\hline 1100.86 & 1098.25 & 1115.08 & 1108.98 & 1103.01 & 1109.36 & 1088.38 & 1087.11 & 1100.87 & 1097.69 & 1088.16 & 1103.16 & 1105.29 \\
\hline 1117.60 & 1114.80 & 1115.92 & 1118.27 & 1114.38 & 1119.04 & 1115.86 & 1113.66 & 1119.05 & 1129.71 & 1112.47 & 1118.62 & 1114.74 \\
\hline 1132.31 & 1131.41 & 1133.89 & 1134.41 & 1134.49 & 1126.26 & 1131.08 & 1129.97 & 1124.29 & 1137.19 & 1136.80 & 1119.92 & 1137.72 \\
\hline 1146.85 & 1146.73 & 1140.70 & 1140.70 & 1137.95 & 1132.92 & 1147.14 & 1137.39 & 1144.94 & 1139.74 & 1146.57 & 1125.23 & 1147.27 \\
\hline 1157.94 & 1156.35 & 1146.55 & 1151.64 & 1143.47 & 1144.60 & 1154.91 & 1154.10 & 1156.40 & 1144.39 & 1152.18 & 1156.33 & 1156.10 \\
\hline 1165.77 & 1158.36 & 1158.27 & 1159.01 & 1153.55 & 1145.70 & 1160.28 & 1158.44 & 1166.18 & 1167.14 & 1157.12 & 1166.52 & 1170.21 \\
\hline 1169.78 & 1172.95 & 1169.91 & 1167.56 & 1166.50 & 1159.24 & 1177.22 & 1176.04 & 1180.04 & 1172.63 & 1175.11 & 1179.43 & 1181.53 \\
\hline 1187.73 & 1184.29 & 1196.87 & 1187.28 & 1178.80 & 1194.12 & 1187.14 & 1179.91 & 1190.26 & 1180.23 & 1179.23 & 1189.05 & 1194.99 \\
\hline 1198.09 & 1198.63 & 1202.37 & 1193.21 & 1191.55 & 1195.92 & 1206.70 & 1206.88 & 1208.62 & 1207.58 & 1198.46 & 1208.92 & 1208.79 \\
\hline
\end{tabular}




\begin{tabular}{|c|c|c|c|c|c|c|c|c|c|c|c|c|}
\hline 1209.71 & 1202.15 & 1209.52 & 1208.38 & 1195.15 & 1198.65 & 1214.05 & 1207.59 & 1210.36 & 1219.11 & 1207.64 & 1210.83 & 1214.00 \\
\hline 1216.21 & 1210.64 & 1211.52 & 1214.65 & 1206.90 & 1207.43 & 1218.51 & 1219.91 & 1217.27 & 1224.29 & 1220.56 & 1218.21 & 1227.72 \\
\hline 1232.08 & 1229.60 & 1222.36 & 1227.36 & 1227.91 & 1221.65 & 1236.52 & 1231.53 & 1233.05 & 1226.00 & 1233.42 & 1237.24 & 1256.80 \\
\hline 1237.52 & 1246.46 & 1260.35 & 1238.79 & 1238.23 & 1242.57 & 1261.62 & 1244.18 & 1256.00 & 1248.56 & 1248.42 & 1257.57 & 1262.08 \\
\hline 1266.19 & 1253.27 & 1267.62 & 1266.66 & 1242.93 & 1261.09 & 1265.68 & 1262.01 & 1261.47 & 1264.30 & 1260.72 & 1262.07 & 1267.22 \\
\hline 1278.47 & 1271.09 & 1274.03 & 1277.63 & 1271.58 & 1268.64 & 1273.88 & 1265.17 & 1266.79 & 1277.48 & 1268.04 & 1267.18 & 1270.30 \\
\hline 1284.57 & 1282.09 & 1280.68 & 1293.25 & 1293.83 & 1285.46 & 1279.24 & 1275.71 & 1277.53 & 1281.04 & 1278.58 & 1276.47 & 1283.36 \\
\hline 1292.23 & 1294.25 & 1323.75 & 1305.24 & 1303.92 & 1321.40 & 1309.28 & 1295.53 & 1299.73 & 1298.72 & 1309.66 & 1307.62 & 1307.33 \\
\hline 1309.26 & 1309.32 & 1325.53 & 1319.68 & 1306.24 & 1327.37 & 1316.26 & 1311.32 & 1308.73 & 1307.17 & 1310.81 & 1315.77 & 1320.79 \\
\hline 1319.70 & 1323.13 & 1331.43 & 1332.84 & 1319.20 & 1337.31 & 1336.25 & 1319.06 & 1319.48 & 1318.58 & 1324.49 & 1327.81 & 1331.79 \\
\hline 1330.40 & 1323.98 & 1344.81 & 1337.55 & 1340.15 & 1341.31 & 1340.58 & 1336.66 & 1339.30 & 1338.14 & 1342.20 & 1341.97 & 1349.27 \\
\hline 1345.68 & 1332.53 & 1349.02 & 1342.39 & 1348.36 & 1347.05 & 1346.45 & 1343.11 & 1344.32 & 1339.60 & 1350.46 & 1346.10 & 1356.65 \\
\hline 1358.07 & 1357.54 & 1365.50 & 1362.68 & 1358.33 & 1358.66 & 1360.95 & 1352.88 & 1352.33 & 1354.35 & 1352.68 & 1352.16 & 1360.45 \\
\hline 1368.07 & 1363.19 & 1368.36 & 1366.75 & 1362.80 & 1372.83 & 1362.24 & 1361.85 & 1362.66 & 1364.94 & 1357.68 & 1361.29 & 1370.91 \\
\hline 1371.54 & 1370.64 & 1373.66 & 1367.84 & 1367.83 & 1375.69 & 1372.72 & 1365.01 & 1364.67 & 1367.31 & 1371.29 & 1366.26 & 1375.09 \\
\hline 1374.62 & 1372.42 & 1375.13 & 1376.45 & 1376.23 & 1380.31 & 1376.66 & 1373.10 & 1371.80 & 1377.05 & 1373.94 & 1372.68 & 1376.21 \\
\hline 1382.05 & 1379.76 & 1377.27 & 1386.95 & 1383.85 & 1385.51 & 1384.89 & 1383.49 & 1379.19 & 1388.54 & 1381.25 & 1380.22 & 1395.94 \\
\hline 1392.26 & 1390.48 & 1392.95 & 1392.20 & 1384.93 & 1393.61 & 1397.51 & 1395.12 & 1384.83 & 1394.55 & 1397.76 & 1390.71 & 1401.40 \\
\hline 1396.09 & 1392.40 & 1400.03 & 1401.73 & 1398.87 & 1402.98 & 1400.32 & 1405.35 & 1390.27 & 1402.74 & 1400.92 & 1410.21 & 1409.46 \\
\hline 1406.14 & 1407.16 & 1404.53 & 1411.89 & 1411.23 & 1406.69 & 1406.58 & 1414.90 & 1417.15 & 1407.87 & 1414.34 & 1418.14 & 1420.07 \\
\hline 1419.94 & 1414.15 & 1410.14 & 1416.84 & 1412.16 & 1412.44 & 1418.63 & 1425.03 & 1420.11 & 1413.99 & 1424.58 & 1443.00 & 1423.27 \\
\hline 1423.57 & 1420.62 & 1415.39 & 1418.03 & 1423.05 & 1414.19 & 1427.54 & 1442.48 & 1443.43 & 1422.02 & 1434.80 & 1447.35 & 1435.08 \\
\hline 1444.80 & 1430.87 & 1431.68 & 1427.16 & 1427.44 & 1419.22 & 1445.27 & 1445.11 & 1453.93 & 1426.39 & 1443.00 & 1455.42 & 1441.08 \\
\hline 1446.64 & 1444.21 & 1444.25 & 1444.24 & 1439.45 & 1432.33 & 1452.94 & 1463.68 & 1460.28 & 1438.67 & 1454.41 & 1461.40 & 1447.01 \\
\hline 1462.83 & 1463.77 & 1446.45 & 1451.10 & 1445.12 & 1445.54 & 1462.33 & 1470.52 & 1461.76 & 1458.88 & 1464.69 & 1482.91 & 1457.00 \\
\hline 1470.60 & 1468.89 & 1465.30 & 1459.39 & 1468.26 & 1469.39 & 1466.69 & 1493.61 & 1483.07 & 1468.24 & 1482.33 & 1490.68 & 1462.23 \\
\hline 1497.22 & 1490.97 & 1471.42 & 1474.19 & 1475.65 & 1470.28 & 1490.60 & 1503.90 & 1503.13 & 1497.43 & 1492.09 & 1503.02 & 1487.06 \\
\hline 1506.00 & 1505.18 & 1501.03 & 1501.35 & 1501.39 & 1499.37 & 1505.18 & 1508.45 & 1503.87 & 1504.84 & 1506.24 & 1504.50 & 1505.99 \\
\hline 1512.36 & 1511.82 & 1505.33 & 1505.05 & 1506.33 & 1506.09 & 1507.58 & 1510.40 & 1510.44 & 1506.65 & 1507.01 & 1511.65 & 1509.69 \\
\hline 1517.16 & 1515.84 & 1506.51 & 1505.84 & 1508.93 & 1510.59 & 1510.67 & 1514.74 & 1512.54 & 1517.97 & 1509.64 & 1512.54 & 1514.96 \\
\hline 1518.30 & 1518.47 & 1510.51 & 1512.59 & 1511.26 & 1511.80 & 1512.10 & 1522.00 & 1526.63 & 1518.23 & 1511.00 & 1526.70 & 1517.65 \\
\hline 1521.72 & 1520.81 & 1517.68 & 1515.92 & 1525.68 & 1517.55 & 1516.63 & 1525.86 & 1531.76 & 1519.53 & 1526.47 & 1532.03 & 1521.31 \\
\hline
\end{tabular}




\begin{tabular}{|c|c|c|c|c|c|c|c|c|c|c|c|c|}
\hline 1529.45 & 1528.60 & 1521.71 & 1521.51 & 1526.13 & 1525.38 & 1527.12 & 1528.78 & 1534.76 & 1527.76 & 1527.40 & 1535.57 & 1525.67 \\
\hline 1531.21 & 1535.08 & 1527.34 & 1526.05 & 1527.67 & 1526.50 & 1528.14 & 1531.36 & 1537.08 & 1529.00 & 1527.98 & 1536.75 & 1537.54 \\
\hline 1538.12 & 1537.25 & 1542.27 & 1531.44 & 1535.31 & 1540.45 & 1531.22 & 1543.34 & 1540.88 & 1536.89 & 1530.27 & 1543.78 & 1548.29 \\
\hline 1542.36 & 1539.27 & 1552.74 & 1548.92 & 1551.33 & 1554.77 & 1551.95 & 1555.21 & 1554.70 & 1548.42 & 1554.07 & 1554.00 & 1551.68 \\
\hline 1551.14 & 1553.28 & 1556.46 & 1550.11 & 1552.89 & 1563.42 & 1555.57 & 1556.17 & 1586.75 & 1561.90 & 1555.81 & 1581.06 & 1561.17 \\
\hline 1612.95 & 1615.51 & 1618.05 & 1614.30 & 1612.95 & 1609.70 & 1620.34 & 1588.68 & 1613.67 & 1625.17 & 1590.26 & 1584.36 & 1612.69 \\
\hline 1628.70 & 1639.97 & 1624.88 & 1624.97 & 1638.26 & 1627.39 & 1628.98 & 1641.06 & 1627.81 & 1626.72 & 1639.06 & 1626.21 & 1618.85 \\
\hline 1630.25 & 1647.02 & 1631.59 & 1634.24 & 1639.49 & 1639.82 & 1632.23 & 1652.53 & 1646.43 & 1635.06 & 1641.01 & 1644.29 & 1627.35 \\
\hline 1656.16 & 1662.11 & 1645.07 & 1649.86 & 1648.72 & 1640.07 & 1660.32 & 1656.70 & 1676.70 & 1668.68 & 1659.28 & 1647.59 & 1649.29 \\
\hline 1664.70 & 1682.42 & 1662.01 & 1672.69 & 1659.91 & 1653.70 & 1673.45 & 1682.91 & 1678.71 & 1676.13 & 1681.44 & 1678.78 & 1664.92 \\
\hline 1678.08 & 1721.47 & 1677.67 & 1679.05 & 1682.92 & 1678.75 & 1678.18 & 1712.20 & 1718.44 & 1677.04 & 1712.85 & 1716.97 & 1677.13 \\
\hline 1721.75 & 1722.93 & 1709.53 & 1712.03 & 1720.90 & 1716.48 & 1714.34 & 1750.04 & 1731.60 & 1743.59 & 1728.64 & 1721.13 & 1690.94 \\
\hline 1739.30 & 1739.69 & 1735.31 & 1733.67 & 1734.57 & 1737.48 & 1766.53 & 1790.76 & 1756.46 & 1796.35 & 1777.41 & 1747.10 & 1782.41 \\
\hline 2550.74 & 2524.11 & 2615.58 & 2658.43 & 2566.47 & 2450.19 & 2981.16 & 2552.01 & 2450.94 & 2640.33 & 2990.02 & 2585.55 & 3006.62 \\
\hline 2982.84 & 2996.79 & 2987.17 & 2938.24 & 2953.75 & 2993.73 & 3025.20 & 2971.16 & 2546.31 & 2971.96 & 3040.26 & 2817.69 & 3060.90 \\
\hline 2988.54 & 2997.20 & 3000.45 & 2988.99 & 2999.40 & 3001.41 & 3026.48 & 3055.68 & 2793.84 & 3026.45 & 3045.39 & 2845.39 & 3077.90 \\
\hline 2992.54 & 3001.21 & 3007.84 & 2999.59 & 3027.16 & 3017.34 & 3073.02 & 3064.82 & 2966.33 & 3055.54 & 3069.69 & 2967.07 & 3083.90 \\
\hline 3023.50 & 3028.23 & 3051.58 & 3007.12 & 3031.96 & 3033.13 & 3077.14 & 3074.05 & 3002.31 & 3060.79 & 3077.59 & 2991.05 & 3087.48 \\
\hline 3057.83 & 3062.19 & 3066.96 & 3022.67 & 3052.08 & 3065.48 & 3081.94 & 3080.43 & 3047.04 & 3063.82 & 3080.97 & 3041.49 & 3090.23 \\
\hline 3061.86 & 3071.06 & 3076.25 & 3052.04 & 3069.69 & 3075.76 & 3097.83 & 3083.44 & 3055.70 & 3082.60 & 3100.00 & 3054.77 & 3094.09 \\
\hline 3076.27 & 3077.20 & 3080.85 & 3062.84 & 3070.87 & 3077.12 & 3110.11 & 3100.44 & 3071.71 & 3083.31 & 3114.25 & 3071.57 & 3100.85 \\
\hline 3094.77 & 3110.63 & 3081.61 & 3067.05 & 3077.92 & 3082.90 & 3123.63 & 3111.36 & 3093.15 & 3096.94 & 3118.74 & 3093.84 & 3123.88 \\
\hline 3105.90 & 3112.30 & 3084.31 & 3076.53 & 3088.08 & 3086.56 & 3151.26 & 3119.06 & 3096.01 & 3110.78 & 3152.87 & 3099.30 & 3156.02 \\
\hline 3108.47 & 3116.89 & 3100.43 & 3083.44 & 3102.90 & 3124.70 & 3159.17 & 3148.64 & 3104.20 & 3125.86 & 3158.23 & 3103.79 & 3161.93 \\
\hline 3114.08 & 3151.14 & 3122.01 & 3102.84 & 3114.58 & 3148.94 & 3174.08 & 3152.86 & 3108.80 & 3152.58 & 3163.98 & 3107.98 & 3174.99 \\
\hline 3149.65 & 3153.36 & 3141.05 & 3113.09 & 3132.22 & 3150.57 & 3179.06 & 3175.32 & 3143.15 & 3163.85 & 3174.40 & 3143.13 & 3186.44 \\
\hline 3150.23 & 3154.79 & 3149.44 & 3143.35 & 3153.49 & 3151.94 & 3189.08 & 3181.71 & 3148.25 & 3177.45 & 3181.87 & 3147.18 & 3197.16 \\
\hline 3154.60 & 3155.60 & 3155.45 & 3150.23 & 3159.86 & 3164.31 & 3193.80 & 3185.36 & 3180.09 & 3178.30 & 3187.93 & 3179.05 & 3221.71 \\
\hline 3157.40 & 3159.73 & 3169.34 & 3158.02 & 3181.20 & 3166.53 & 3208.49 & 3185.79 & 3181.47 & 3192.11 & 3190.41 & 3180.64 & 3225.57 \\
\hline 3192.26 & 3189.28 & 3194.14 & 3193.93 & 3192.07 & 3191.19 & 3222.70 & 3200.24 & 3191.35 & 3216.63 & 3212.05 & 3191.19 & 3228.17 \\
\hline 3223.10 & 3220.26 & 3224.32 & 3224.65 & 3222.67 & 3221.92 & 3223.99 & 3213.86 & 3201.03 & 3222.90 & 3221.18 & 3200.35 & 3241.98 \\
\hline 3237.54 & 3234.43 & 3245.22 & 3242.53 & 3238.68 & 3237.90 & 3240.97 & 3217.05 & 3211.37 & 3234.58 & 3238.62 & 3211.31 & 3243.76 \\
\hline
\end{tabular}




\begin{tabular}{|c|c|c|c|c|c|c|c|c|c|c|c|c|}
\hline 3306.80 & 3236.06 & 3311.46 & 3333.35 & 3348.71 & 3511.23 & 3324.21 & 3234.29 & 3213.82 & 3290.41 & 3243.27 & 3213.17 & 3272.78 \\
\hline 3355.23 & 3487.89 & 3509.08 & 3497.98 & 3520.39 & 3541.12 & 3496.83 & 3483.44 & 3228.30 & 3524.71 & 3526.78 & 3227.67 & 3407.72 \\
\hline 3481.67 & 3615.44 & 3576.79 & 3684.34 & 3685.92 & 3676.75 & 3649.11 & 3572.00 & 3470.61 & 3669.86 & 3660.12 & 3380.74 & 3506.03 \\
\hline 3678.82 & 3679.16 & 3685.90 & 3697.06 & 3697.81 & 3683.41 & 3689.82 & 3694.21 & 3661.89 & 3715.92 & 3708.89 & 3656.42 & 3660.51 \\
\hline 3707.43 & 3697.68 & 3734.09 & 3708.18 & 3725.55 & 3725.68 & 3715.13 & 3705.90 & 3673.97 & 3742.10 & 3721.06 & 3662.39 & 3744.66 \\
\hline
\end{tabular}


Table $S 4$ continued:

\begin{tabular}{|c|c|c|c|c|c|c|c|c|c|c|c|c|}
\hline Z8 & Z9 & Z10 & Z11 & Z12 & Z13 & Z14 & Z15 & Z16 & Z17 & Z18 & Z19 & $\mathbf{Z 2 0}$ \\
\hline 22.00 & 26.89 & 19.37 & 21.02 & 24.98 & 27.16 & 28.57 & 29.36 & 26.30 & 21.90 & 29.07 & 25.43 & 16.72 \\
\hline 34.06 & 37.25 & 32.30 & 33.75 & 34.46 & 32.53 & 38.28 & 34.46 & 32.81 & 30.28 & 34.12 & 33.05 & 27.81 \\
\hline 49.59 & 44.49 & 38.83 & 35.06 & 48.56 & 43.96 & 45.73 & 48.55 & 44.46 & 42.75 & 47.56 & 49.02 & 32.47 \\
\hline 55.68 & 59.42 & 54.01 & 53.48 & 57.75 & 59.99 & 58.87 & 60.23 & 56.95 & 61.30 & 70.88 & 68.07 & 36.04 \\
\hline 71.65 & 71.90 & 70.39 & 74.66 & 69.46 & 74.87 & 72.32 & 73.27 & 79.07 & 77.62 & 76.78 & 71.85 & 58.17 \\
\hline 79.06 & 73.07 & 84.60 & 87.21 & 84.14 & 94.06 & 73.90 & 80.79 & 87.75 & 82.87 & 95.86 & 90.90 & 64.66 \\
\hline 98.12 & 96.22 & 95.92 & 97.03 & 95.90 & 112.54 & 89.96 & 99.45 & 104.07 & 97.78 & 100.71 & 98.47 & 93.16 \\
\hline 106.26 & 121.33 & 102.01 & 102.31 & 99.53 & 116.63 & 120.36 & 119.20 & 113.90 & 106.12 & 117.77 & 104.14 & 100.04 \\
\hline 127.81 & 128.61 & 118.96 & 126.89 & 104.13 & 135.12 & 126.65 & 128.82 & 142.51 & 146.93 & 136.55 & 131.91 & 100.66 \\
\hline 135.37 & 141.75 & 130.83 & 135.73 & 133.72 & 148.69 & 140.96 & 146.52 & 152.11 & 152.37 & 150.37 & 144.89 & 130.58 \\
\hline 149.22 & 161.11 & 150.54 & 152.15 & 154.13 & 158.06 & 158.77 & 162.37 & 159.66 & 156.87 & 154.23 & 157.02 & 149.96 \\
\hline 164.51 & 168.96 & 156.81 & 158.23 & 167.95 & 179.72 & 168.74 & 175.48 & 181.74 & 180.10 & 177.56 & 175.42 & 156.19 \\
\hline 174.45 & 171.55 & 164.70 & 167.16 & 177.72 & 186.81 & 169.94 & 180.67 & 187.22 & 184.92 & 182.60 & 179.97 & 172.52 \\
\hline 195.97 & 188.94 & 192.59 & 186.89 & 189.44 & 192.91 & 189.33 & 196.91 & 189.35 & 197.82 & 187.54 & 181.95 & 181.23 \\
\hline 210.53 & 206.39 & 194.20 & 191.91 & 195.66 & 204.11 & 205.48 & 198.62 & 196.89 & 204.08 & 203.98 & 203.82 & 201.26 \\
\hline 224.58 & 214.10 & 214.98 & 198.06 & 198.26 & 209.75 & 212.47 & 217.28 & 213.90 & 217.22 & 212.15 & 205.07 & 205.27 \\
\hline 234.48 & 229.75 & 223.56 & 218.46 & 219.51 & 219.57 & 232.63 & 224.75 & 225.69 & 227.05 & 235.62 & 227.04 & 219.81 \\
\hline 239.56 & 236.76 & 230.90 & 229.34 & 224.65 & 227.31 & 238.06 & 231.29 & 231.86 & 230.69 & 239.13 & 233.82 & 223.79 \\
\hline 252.89 & 241.78 & 238.72 & 236.14 & 233.86 & 234.55 & 241.97 & 235.51 & 238.50 & 236.73 & 244.75 & 242.96 & 233.34 \\
\hline 258.47 & 247.64 & 240.56 & 242.80 & 247.72 & 239.72 & 249.56 & 242.39 & 242.81 & 243.08 & 253.78 & 252.64 & 238.56 \\
\hline 276.20 & 255.78 & 263.10 & 247.86 & 263.26 & 245.03 & 257.10 & 243.39 & 258.85 & 268.73 & 264.84 & 259.25 & 253.78 \\
\hline 290.86 & 267.21 & 269.09 & 268.48 & 282.96 & 259.37 & 269.46 & 264.33 & 282.69 & 277.53 & 271.26 & 269.38 & 258.67 \\
\hline 295.22 & 272.31 & 286.39 & 286.73 & 287.27 & 281.85 & 281.06 & 280.33 & 285.75 & 284.02 & 288.12 & 281.76 & 272.63 \\
\hline 304.75 & 286.47 & 306.14 & 306.01 & 305.87 & 289.76 & 292.12 & 283.45 & 300.34 & 285.87 & 293.26 & 289.50 & 281.36 \\
\hline 310.47 & 300.20 & 309.99 & 310.30 & 310.30 & 304.96 & 310.94 & 307.95 & 312.55 & 301.81 & 300.90 & 291.26 & 300.10 \\
\hline 323.76 & 313.85 & 323.15 & 323.62 & 327.58 & 313.97 & 314.57 & 318.11 & 319.11 & 319.17 & 308.28 & 305.81 & 310.54 \\
\hline 333.99 & 320.58 & 329.34 & 329.58 & 329.66 & 329.99 & 323.30 & 322.95 & 335.43 & 325.94 & 328.56 & 327.50 & 320.87 \\
\hline 359.79 & 338.34 & 331.90 & 338.83 & 344.95 & 336.77 & 340.55 & 330.55 & 347.59 & 339.54 & 343.51 & 331.08 & 324.18 \\
\hline 367.32 & 345.98 & 358.84 & 360.19 & 356.11 & 346.65 & 355.36 & 346.10 & 362.98 & 343.86 & 349.21 & 346.83 & 338.90 \\
\hline 370.59 & 353.67 & 368.20 & 372.32 & 358.92 & 364.68 & 361.23 & 360.87 & 367.36 & 358.98 & 358.81 & 359.14 & 353.16 \\
\hline 372.77 & 358.51 & 371.06 & 389.68 & 368.75 & 366.70 & 361.75 & 370.18 & 375.10 & 363.79 & 364.96 & 363.56 & 371.30 \\
\hline 385.19 & 377.09 & 391.92 & 394.83 & 375.43 & 373.88 & 379.81 & 379.53 & 376.75 & 378.29 & 372.60 & 365.15 & 378.66 \\
\hline
\end{tabular}




\begin{tabular}{|c|c|c|c|c|c|c|c|c|c|c|c|c|}
\hline 416.29 & 388.12 & 417.35 & 425.44 & 386.17 & 395.06 & 389.16 & 390.87 & 386.87 & 384.60 & 383.01 & 374.76 & 382.79 \\
\hline 423.19 & 414.85 & 431.41 & 435.60 & 399.50 & 407.29 & 414.43 & 406.00 & 389.66 & 400.66 & 407.69 & 380.51 & 395.24 \\
\hline 429.71 & 429.39 & 436.67 & 446.40 & 427.91 & 422.67 & 424.75 & 427.47 & 396.15 & 427.04 & 421.39 & 400.71 & 422.83 \\
\hline 445.44 & 453.29 & 456.88 & 456.43 & 435.48 & 430.35 & 435.14 & 432.66 & 409.91 & 441.01 & 429.61 & 408.31 & 439.64 \\
\hline 450.70 & 461.38 & 459.83 & 466.69 & 442.29 & 446.96 & 457.73 & 447.70 & 426.29 & 451.34 & 435.85 & 431.53 & 443.96 \\
\hline 456.13 & 469.52 & 470.78 & 482.18 & 463.24 & 453.88 & 461.82 & 454.48 & 431.37 & 456.34 & 448.33 & 434.66 & 458.09 \\
\hline 459.67 & 481.10 & 482.42 & 487.92 & 466.34 & 463.34 & 469.44 & 470.08 & 447.03 & 458.12 & 463.73 & 448.09 & 466.32 \\
\hline 482.75 & 486.75 & 487.20 & 494.43 & 486.22 & 468.42 & 492.67 & 477.37 & 455.88 & 484.18 & 472.04 & 452.47 & 492.30 \\
\hline 483.68 & 494.54 & 493.14 & 499.34 & 490.84 & 478.53 & 498.97 & 492.60 & 476.35 & 490.02 & 486.62 & 469.52 & 496.80 \\
\hline 493.48 & 502.16 & 495.90 & 504.33 & 499.20 & 486.99 & 501.27 & 498.03 & 486.87 & 494.12 & 496.37 & 479.51 & 503.63 \\
\hline 503.02 & 507.28 & 505.76 & 509.19 & 506.91 & 492.85 & 507.22 & 510.68 & 494.00 & 501.40 & 501.64 & 495.57 & 507.88 \\
\hline 525.60 & 520.08 & 526.45 & 523.58 & 520.87 & 499.32 & 516.86 & 528.07 & 498.42 & 522.12 & 503.90 & 501.81 & 517.94 \\
\hline 539.22 & 525.72 & 532.07 & 534.09 & 538.20 & 501.52 & 529.27 & 537.83 & 502.43 & 533.24 & 505.31 & 502.71 & 520.72 \\
\hline 545.25 & 541.45 & 543.83 & 543.75 & 540.38 & 517.61 & 540.27 & 549.18 & 518.46 & 544.98 & 522.31 & 519.92 & 541.33 \\
\hline 572.38 & 573.66 & 568.72 & 567.60 & 554.22 & 536.63 & 574.12 & 584.66 & 537.47 & 557.11 & 540.52 & 540.01 & 548.51 \\
\hline 598.71 & 587.43 & 587.94 & 584.51 & 568.12 & 555.42 & 588.16 & 588.46 & 553.53 & 580.86 & 553.95 & 549.44 & 566.71 \\
\hline 604.24 & 602.57 & 603.33 & 596.49 & 582.65 & 584.30 & 601.93 & 602.72 & 587.53 & 593.79 & 583.20 & 589.49 & 584.87 \\
\hline 639.89 & 612.39 & 614.78 & 604.08 & 601.22 & 603.64 & 612.04 & 631.10 & 625.19 & 608.46 & 596.59 & 608.49 & 601.21 \\
\hline 642.36 & 631.56 & 643.34 & 643.88 & 627.76 & 633.69 & 630.70 & 640.22 & 635.66 & 633.19 & 620.00 & 618.33 & 618.63 \\
\hline 656.32 & 641.99 & 666.29 & 664.18 & 634.44 & 644.42 & 640.28 & 649.84 & 644.86 & 650.80 & 627.76 & 637.96 & 626.17 \\
\hline 683.12 & 646.64 & 682.76 & 683.61 & 640.02 & 654.44 & 645.00 & 652.44 & 653.46 & 658.65 & 645.82 & 644.15 & 645.99 \\
\hline 699.08 & 666.81 & 705.98 & 709.39 & 650.69 & 675.70 & 650.06 & 664.16 & 675.05 & 664.15 & 676.28 & 674.56 & 652.85 \\
\hline 713.94 & 688.81 & 710.35 & 715.02 & 659.34 & 681.47 & 668.53 & 670.03 & 678.17 & 682.52 & 696.94 & 690.78 & 671.92 \\
\hline 715.97 & 700.68 & 716.77 & 719.13 & 683.90 & 701.00 & 675.82 & 678.57 & 704.06 & 690.95 & 708.10 & 702.22 & 699.47 \\
\hline 732.38 & 706.56 & 726.01 & 731.39 & 693.11 & 719.79 & 687.32 & 689.03 & 712.07 & 697.17 & 719.46 & 710.62 & 708.16 \\
\hline 746.82 & 737.05 & 747.38 & 750.24 & 714.13 & 748.33 & 704.42 & 698.22 & 725.03 & 729.72 & 735.51 & 716.81 & 717.11 \\
\hline 750.32 & 755.35 & 769.43 & 780.83 & 721.42 & 755.33 & 742.89 & 741.38 & 750.10 & 759.92 & 760.06 & 740.14 & 743.93 \\
\hline 760.03 & 773.16 & 782.01 & 786.83 & 743.14 & 763.65 & 761.37 & 760.68 & 758.77 & 769.74 & 768.70 & 765.86 & 755.75 \\
\hline 792.76 & 778.84 & 790.81 & 793.05 & 756.60 & 781.00 & 769.12 & 765.71 & 773.69 & 779.13 & 780.90 & 771.51 & 777.05 \\
\hline 798.98 & 794.41 & 796.02 & 801.15 & 766.10 & 787.81 & 777.38 & 780.35 & 789.55 & 799.28 & 792.41 & 790.69 & 785.17 \\
\hline 801.44 & 797.34 & 805.92 & 815.06 & 789.52 & 800.97 & 796.33 & 801.23 & 797.64 & 801.14 & 797.53 & 791.56 & 799.84 \\
\hline 822.89 & 801.42 & 821.90 & 822.12 & 799.87 & 814.77 & 803.88 & 804.56 & 814.64 & 815.90 & 803.85 & 792.96 & 802.27 \\
\hline 855.57 & 817.60 & 829.17 & 846.98 & 809.80 & 829.14 & 804.63 & 811.12 & 822.89 & 825.73 & 823.47 & 822.23 & 818.38 \\
\hline 867.22 & 828.82 & 850.86 & 862.32 & 820.32 & 840.46 & 825.12 & 821.84 & 832.57 & 835.80 & 853.56 & 854.09 & 842.21 \\
\hline 877.16 & 859.41 & 869.36 & 867.51 & 857.58 & 845.69 & 856.67 & 831.56 & 839.80 & 852.64 & 860.71 & 859.63 & 849.47 \\
\hline
\end{tabular}




\begin{tabular}{|c|c|c|c|c|c|c|c|c|c|c|c|c|}
\hline 884.29 & 875.91 & 881.56 & 872.85 & 869.24 & 878.99 & 874.29 & 867.25 & 875.82 & 866.63 & 881.46 & 881.34 & 875.01 \\
\hline 910.19 & 887.05 & 890.03 & 881.55 & 879.99 & 889.86 & 887.01 & 881.52 & 887.62 & 878.18 & 896.52 & 887.20 & 876.20 \\
\hline 922.49 & 899.05 & 916.90 & 909.80 & 901.70 & 904.48 & 901.46 & 886.57 & 893.59 & 881.53 & 897.43 & 923.88 & 878.48 \\
\hline 944.84 & 923.72 & 923.54 & 925.52 & 910.73 & 941.64 & 929.01 & 903.10 & 942.33 & 883.89 & 925.68 & 936.39 & 891.97 \\
\hline 949.90 & 930.21 & 941.43 & 937.33 & 941.44 & 948.37 & 936.02 & 928.17 & 945.62 & 896.56 & 944.28 & 944.49 & 940.58 \\
\hline 959.95 & 938.43 & 958.86 & 945.05 & 952.66 & 952.39 & 957.64 & 942.74 & 961.11 & 929.39 & 946.94 & 950.90 & 943.13 \\
\hline 969.93 & 958.76 & 965.37 & 962.42 & 959.55 & 964.16 & 959.18 & 967.50 & 968.99 & 944.26 & 951.51 & 966.50 & 965.67 \\
\hline 974.32 & 963.51 & 974.63 & 965.11 & 965.02 & 975.98 & 966.88 & 980.01 & 970.51 & 969.97 & 964.54 & 970.26 & 975.87 \\
\hline 997.76 & 969.25 & 996.23 & 975.24 & 969.63 & 987.07 & 983.37 & 983.44 & 980.09 & 981.09 & 970.68 & 980.09 & 978.96 \\
\hline 1003.78 & 986.59 & 1014.65 & 1016.54 & 984.35 & 987.90 & 986.47 & 996.76 & 1006.52 & 984.01 & 983.92 & 997.81 & 991.56 \\
\hline 1024.47 & 999.68 & 1018.26 & 1019.42 & 1000.74 & 1009.05 & 995.48 & 997.89 & 1011.30 & 1001.13 & 1008.98 & 1017.30 & 1001.66 \\
\hline 1041.96 & 1014.03 & 1053.22 & 1022.93 & 1021.92 & 1016.52 & 1014.05 & 1003.64 & 1035.61 & 1004.56 & 1019.53 & 1031.86 & 1034.83 \\
\hline 1058.75 & 1043.91 & 1055.83 & 1055.92 & 1041.29 & 1042.87 & 1045.52 & 1027.00 & 1041.43 & 1031.20 & 1036.74 & 1047.85 & 1043.10 \\
\hline 1063.42 & 1052.96 & 1062.68 & 1060.78 & 1046.94 & 1069.51 & 1055.01 & 1057.77 & 1069.84 & 1060.52 & 1058.97 & 1061.53 & 1055.85 \\
\hline 1076.12 & 1082.38 & 1069.01 & 1066.07 & 1062.19 & 1074.03 & 1081.86 & 1066.48 & 1074.07 & 1067.96 & 1079.23 & 1079.72 & 1076.42 \\
\hline 1083.38 & 1096.38 & 1077.55 & 1070.97 & 1073.87 & 1076.04 & 1092.08 & 1081.34 & 1079.93 & 1076.19 & 1088.04 & 1087.61 & 1081.59 \\
\hline 1088.70 & 1108.06 & 1090.47 & 1089.63 & 1086.79 & 1085.44 & 1105.15 & 1101.35 & 1086.47 & 1091.90 & 1091.51 & 1089.48 & 1086.43 \\
\hline 1100.24 & 1112.47 & 1101.50 & 1098.66 & 1087.72 & 1098.12 & 1116.75 & 1106.76 & 1104.20 & 1100.55 & 1100.95 & 1105.23 & 1098.34 \\
\hline 1120.17 & 1118.27 & 1120.35 & 1120.01 & 1111.23 & 1115.15 & 1120.16 & 1116.29 & 1115.89 & 1108.54 & 1117.34 & 1117.31 & 1130.67 \\
\hline 1122.74 & 1139.14 & 1133.21 & 1120.80 & 1135.85 & 1126.55 & 1137.26 & 1141.10 & 1127.91 & 1125.75 & 1125.35 & 1119.58 & 1135.01 \\
\hline 1144.31 & 1149.55 & 1147.15 & 1136.37 & 1147.39 & 1136.55 & 1149.19 & 1147.93 & 1139.16 & 1143.88 & 1126.95 & 1139.10 & 1138.99 \\
\hline 1155.87 & 1159.59 & 1154.49 & 1153.67 & 1155.46 & 1140.63 & 1160.03 & 1156.59 & 1141.71 & 1155.90 & 1148.75 & 1147.91 & 1151.08 \\
\hline 1172.77 & 1171.62 & 1165.84 & 1165.05 & 1160.85 & 1157.49 & 1169.33 & 1170.82 & 1156.00 & 1163.35 & 1167.85 & 1167.24 & 1156.43 \\
\hline 1180.33 & 1188.85 & 1174.37 & 1174.71 & 1177.77 & 1177.89 & 1174.47 & 1180.47 & 1180.56 & 1181.40 & 1181.05 & 1179.73 & 1169.07 \\
\hline 1187.53 & 1190.95 & 1178.83 & 1180.34 & 1185.51 & 1194.78 & 1187.22 & 1193.12 & 1194.43 & 1195.31 & 1192.94 & 1194.02 & 1177.16 \\
\hline 1208.64 & 1204.18 & 1203.31 & 1207.26 & 1202.42 & 1203.68 & 1201.83 & 1200.39 & 1200.21 & 1207.12 & 1203.88 & 1204.42 & 1198.72 \\
\hline 1211.25 & 1211.44 & 1213.32 & 1213.41 & 1209.10 & 1208.79 & 1207.51 & 1211.34 & 1213.43 & 1210.78 & 1211.20 & 1210.95 & 1209.35 \\
\hline 1220.06 & 1218.36 & 1217.16 & 1221.65 & 1215.15 & 1220.34 & 1211.56 & 1220.33 & 1217.43 & 1221.18 & 1226.50 & 1223.97 & 1227.22 \\
\hline 1242.00 & 1234.38 & 1234.10 & 1237.97 & 1232.59 & 1243.93 & 1228.23 & 1247.78 & 1249.88 & 1249.01 & 1251.07 & 1255.74 & 1239.10 \\
\hline 1255.51 & 1263.79 & 1258.17 & 1259.41 & 1258.46 & 1245.24 & 1252.78 & 1256.55 & 1256.51 & 1258.45 & 1257.78 & 1257.20 & 1244.42 \\
\hline 1262.03 & 1271.81 & 1260.11 & 1264.68 & 1262.07 & 1256.49 & 1269.71 & 1260.22 & 1261.82 & 1263.31 & 1265.60 & 1266.22 & 1253.30 \\
\hline 1270.00 & 1274.61 & 1271.27 & 1272.74 & 1270.78 & 1276.06 & 1276.03 & 1270.68 & 1274.40 & 1270.41 & 1270.56 & 1271.31 & 1270.02 \\
\hline 1285.68 & 1293.89 & 1273.50 & 1281.23 & 1283.34 & 1289.15 & 1289.71 & 1288.60 & 1289.37 & 1275.15 & 1289.20 & 1294.36 & 1293.69 \\
\hline 1311.17 & 1308.11 & 1284.40 & 1303.43 & 1306.59 & 1307.81 & 1301.76 & 1305.53 & 1311.56 & 1305.52 & 1308.71 & 1310.19 & 1299.09 \\
\hline 1324.09 & 1317.98 & 1306.68 & 1314.70 & 1314.90 & 1315.16 & 1312.04 & 1314.23 & 1322.53 & 1327.19 & 1318.16 & 1319.40 & 1311.52 \\
\hline
\end{tabular}




\begin{tabular}{|c|c|c|c|c|c|c|c|c|c|c|c|c|}
\hline 1331.43 & 1324.79 & 1319.32 & 1329.55 & 1335.47 & 1327.21 & 1323.00 & 1322.41 & 1336.39 & 1337.79 & 1319.24 & 1335.35 & 1318.58 \\
\hline 1340.22 & 1335.36 & 1332.11 & 1342.96 & 1339.51 & 1343.40 & 1330.62 & 1344.33 & 1341.14 & 1342.48 & 1340.94 & 1342.37 & 1334.99 \\
\hline 1351.60 & 1348.85 & 1342.43 & 1344.86 & 1344.41 & 1346.13 & 1346.88 & 1354.38 & 1353.57 & 1354.23 & 1354.60 & 1358.92 & 1344.79 \\
\hline 1360.56 & 1358.06 & 1354.51 & 1357.91 & 1360.06 & 1358.28 & 1360.00 & 1358.96 & 1359.71 & 1360.97 & 1357.60 & 1365.63 & 1360.94 \\
\hline 1364.32 & 1362.88 & 1362.30 & 1362.61 & 1367.89 & 1363.89 & 1361.33 & 1360.46 & 1369.48 & 1370.15 & 1364.16 & 1369.33 & 1364.81 \\
\hline 1373.37 & 1369.27 & 1372.04 & 1375.57 & 1369.56 & 1372.23 & 1370.57 & 1372.55 & 1377.94 & 1375.64 & 1370.86 & 1373.52 & 1367.67 \\
\hline 1377.45 & 1376.10 & 1380.24 & 1382.03 & 1375.94 & 1380.11 & 1374.50 & 1375.58 & 1382.30 & 1383.17 & 1389.54 & 1383.97 & 1372.18 \\
\hline 1382.11 & 1380.91 & 1387.14 & 1391.29 & 1380.24 & 1400.02 & 1376.25 & 1379.78 & 1389.35 & 1391.76 & 1393.77 & 1389.87 & 1380.64 \\
\hline 1391.15 & 1395.34 & 1394.46 & 1394.98 & 1389.26 & 1411.65 & 1383.68 & 1397.09 & 1411.04 & 1401.38 & 1403.60 & 1403.74 & 1395.92 \\
\hline 1413.71 & 1404.65 & 1406.20 & 1416.47 & 1395.61 & 1416.00 & 1399.89 & 1407.64 & 1419.82 & 1409.84 & 1415.08 & 1414.81 & 1398.11 \\
\hline 1417.44 & 1411.18 & 1415.80 & 1434.31 & 1404.59 & 1429.89 & 1409.03 & 1410.29 & 1423.09 & 1415.68 & 1419.44 & 1421.10 & 1409.04 \\
\hline 1419.12 & 1419.95 & 1434.99 & 1442.52 & 1410.13 & 1437.49 & 1413.02 & 1421.78 & 1427.38 & 1425.34 & 1437.47 & 1427.44 & 1417.24 \\
\hline 1443.44 & 1431.27 & 1441.14 & 1450.96 & 1427.59 & 1442.66 & 1425.89 & 1424.70 & 1439.96 & 1433.90 & 1440.06 & 1437.61 & 1427.19 \\
\hline 1447.91 & 1443.35 & 1451.34 & 1456.72 & 1443.27 & 1445.56 & 1433.59 & 1439.65 & 1443.69 & 1440.83 & 1458.56 & 1439.82 & 1432.48 \\
\hline 1461.05 & 1444.11 & 1452.32 & 1463.74 & 1444.77 & 1456.07 & 1441.84 & 1447.26 & 1452.53 & 1445.82 & 1464.72 & 1453.34 & 1436.85 \\
\hline 1473.66 & 1457.49 & 1457.43 & 1470.02 & 1462.55 & 1476.36 & 1456.33 & 1452.43 & 1466.30 & 1462.58 & 1469.53 & 1466.57 & 1439.72 \\
\hline 1484.85 & 1469.26 & 1465.95 & 1480.26 & 1467.52 & 1495.53 & 1468.95 & 1460.57 & 1480.27 & 1480.25 & 1498.61 & 1491.64 & 1465.51 \\
\hline 1503.42 & 1502.29 & 1470.30 & 1488.68 & 1491.08 & 1498.61 & 1502.14 & 1488.62 & 1500.93 & 1491.89 & 1500.61 & 1501.88 & 1489.81 \\
\hline 1504.53 & 1506.38 & 1496.57 & 1501.46 & 1503.94 & 1501.59 & 1504.09 & 1503.16 & 1501.18 & 1505.94 & 1504.61 & 1504.57 & 1499.72 \\
\hline 1510.07 & 1512.86 & 1500.80 & 1502.73 & 1506.79 & 1519.61 & 1513.23 & 1510.06 & 1516.90 & 1514.11 & 1512.91 & 1508.56 & 1508.15 \\
\hline 1512.86 & 1515.17 & 1503.84 & 1504.13 & 1510.40 & 1524.42 & 1514.94 & 1516.46 & 1521.21 & 1517.92 & 1516.14 & 1515.92 & 1510.42 \\
\hline 1526.64 & 1519.56 & 1510.98 & 1510.99 & 1514.66 & 1529.61 & 1522.83 & 1518.29 & 1529.39 & 1522.09 & 1521.29 & 1519.79 & 1517.41 \\
\hline 1531.33 & 1522.50 & 1521.12 & 1525.33 & 1526.97 & 1531.73 & 1526.78 & 1525.69 & 1530.98 & 1528.10 & 1526.43 & 1525.97 & 1526.59 \\
\hline 1534.70 & 1526.02 & 1527.37 & 1529.71 & 1527.50 & 1533.19 & 1535.10 & 1534.49 & 1532.25 & 1530.09 & 1528.98 & 1528.82 & 1529.06 \\
\hline 1535.78 & 1537.77 & 1531.35 & 1530.10 & 1531.21 & 1537.70 & 1538.12 & 1538.39 & 1548.32 & 1536.89 & 1538.07 & 1536.29 & 1530.21 \\
\hline 1545.39 & 1545.04 & 1533.95 & 1531.30 & 1532.95 & 1543.43 & 1546.17 & 1550.34 & 1549.89 & 1545.72 & 1546.47 & 1547.86 & 1533.70 \\
\hline 1554.54 & 1548.06 & 1536.80 & 1533.88 & 1552.44 & 1551.14 & 1548.97 & 1552.75 & 1558.33 & 1554.74 & 1549.85 & 1552.65 & 1550.73 \\
\hline 1576.09 & 1588.09 & 1552.08 & 1553.14 & 1556.56 & 1575.76 & 1589.02 & 1561.56 & 1585.16 & 1568.83 & 1555.59 & 1559.96 & 1551.59 \\
\hline 1617.86 & 1621.23 & 1615.91 & 1566.60 & 1619.26 & 1596.34 & 1623.20 & 1611.81 & 1613.40 & 1578.56 & 1570.87 & 1608.99 & 1611.23 \\
\hline 1626.16 & 1626.64 & 1626.71 & 1623.67 & 1626.50 & 1611.52 & 1627.84 & 1628.76 & 1617.88 & 1630.43 & 1607.83 & 1621.85 & 1639.59 \\
\hline 1627.73 & 1628.27 & 1647.58 & 1641.81 & 1650.91 & 1642.98 & 1660.29 & 1657.93 & 1628.60 & 1641.14 & 1644.75 & 1632.06 & 1643.25 \\
\hline 1646.15 & 1654.56 & 1678.54 & 1647.43 & 1660.30 & 1651.06 & 1667.93 & 1661.09 & 1649.29 & 1652.70 & 1648.39 & 1647.35 & 1663.04 \\
\hline 1678.30 & 1676.88 & 1693.04 & 1677.82 & 1669.86 & 1670.08 & 1676.79 & 1673.61 & 1673.78 & 1676.17 & 1668.97 & 1669.77 & 1671.49 \\
\hline 1697.70 & 1678.58 & 1717.97 & 1714.29 & 1679.38 & 1700.51 & 1679.87 & 1680.03 & 1687.07 & 1706.88 & 1695.65 & 1679.78 & 1684.81 \\
\hline 1719.74 & 1695.57 & 1731.61 & 1723.73 & 1712.42 & 1718.08 & 1695.16 & 1690.54 & 1713.46 & 1709.51 & 1717.48 & 1696.84 & 1719.75 \\
\hline
\end{tabular}




\begin{tabular}{|c|c|c|c|c|c|c|c|c|c|c|c|c|}
\hline 1736.09 & 1789.46 & 1761.24 & 1752.90 & 1765.28 & 1744.79 & 1788.98 & 1782.06 & 1736.28 & 1788.55 & 1748.63 & 1740.23 & 1792.31 \\
\hline 2558.37 & 2319.68 & 2329.37 & 2949.12 & 2743.77 & 2687.64 & 2272.41 & 2540.82 & 2585.82 & 2995.61 & 2871.99 & 2560.48 & 2924.57 \\
\hline 2609.18 & 3019.56 & 2940.18 & 2953.97 & 2974.85 & 2928.42 & 2631.14 & 3006.20 & 2805.40 & 3056.31 & 2996.93 & 2905.37 & 2982.04 \\
\hline 2898.78 & 3053.89 & 2951.58 & 3012.99 & 3020.56 & 2982.44 & 3018.19 & 3055.99 & 2962.74 & 3078.38 & 3029.14 & 2990.49 & 3024.71 \\
\hline 2966.08 & 3068.21 & 2963.37 & 3034.17 & 3074.30 & 3015.76 & 3062.81 & 3075.61 & 2970.22 & 3089.00 & 3062.70 & 3031.39 & 3055.01 \\
\hline 3014.44 & 3080.06 & 3036.29 & 3038.62 & 3077.96 & 3022.11 & 3078.57 & 3083.62 & 3024.53 & 3090.53 & 3068.97 & 3032.96 & 3062.12 \\
\hline 3047.86 & 3080.42 & 3048.31 & 3048.42 & 3083.12 & 3039.60 & 3079.54 & 3086.77 & 3062.33 & 3096.71 & 3070.23 & 3071.25 & 3064.04 \\
\hline 3057.64 & 3092.16 & 3054.40 & 3052.43 & 3097.58 & 3069.28 & 3083.87 & 3091.89 & 3077.23 & 3098.93 & 3074.22 & 3075.20 & 3089.04 \\
\hline 3072.32 & 3093.35 & 3069.19 & 3068.99 & 3108.82 & 3075.85 & 3092.32 & 3100.82 & 3086.76 & 3103.77 & 3084.75 & 3079.49 & 3105.29 \\
\hline 3095.18 & 3097.11 & 3083.94 & 3079.78 & 3125.77 & 3098.73 & 3096.30 & 3125.28 & 3100.25 & 3117.85 & 3100.73 & 3089.11 & 3105.87 \\
\hline 3102.99 & 3133.68 & 3097.98 & 3096.27 & 3151.03 & 3104.52 & 3132.74 & 3156.77 & 3105.90 & 3149.39 & 3112.60 & 3102.19 & 3116.37 \\
\hline 3106.25 & 3153.87 & 3102.02 & 3108.98 & 3160.30 & 3126.87 & 3153.45 & 3161.05 & 3126.22 & 3165.59 & 3122.45 & 3127.17 & 3128.97 \\
\hline 3111.06 & 3156.23 & 3140.62 & 3140.38 & 3175.00 & 3141.88 & 3154.95 & 3174.12 & 3149.55 & 3177.44 & 3142.62 & 3143.91 & 3132.43 \\
\hline 3144.30 & 3165.25 & 3146.71 & 3144.92 & 3178.91 & 3146.68 & 3164.61 & 3186.59 & 3158.08 & 3180.32 & 3150.47 & 3151.23 & 3152.89 \\
\hline 3149.02 & 3176.76 & 3179.38 & 3178.43 & 3189.96 & 3181.03 & 3176.00 & 3199.94 & 3180.38 & 3191.35 & 3168.52 & 3169.49 & 3173.51 \\
\hline 3180.78 & 3181.70 & 3186.95 & 3185.79 & 3196.49 & 3182.92 & 3177.79 & 3221.70 & 3185.91 & 3193.59 & 3176.28 & 3175.67 & 3182.60 \\
\hline 3182.28 & 3192.74 & 3199.80 & 3193.42 & 3209.07 & 3187.09 & 3191.97 & 3226.28 & 3190.44 & 3222.58 & 3180.71 & 3182.31 & 3190.00 \\
\hline 3192.28 & 3195.35 & 3206.05 & 3211.24 & 3223.30 & 3213.41 & 3197.77 & 3226.45 & 3215.27 & 3228.13 & 3185.84 & 3185.51 & 3196.91 \\
\hline 3201.25 & 3224.93 & 3212.82 & 3212.04 & 3224.91 & 3214.49 & 3225.29 & 3242.12 & 3217.37 & 3233.58 & 3212.20 & 3210.64 & 3221.15 \\
\hline 3211.31 & 3241.66 & 3213.49 & 3226.19 & 3242.47 & 3220.70 & 3242.47 & 3244.40 & 3235.83 & 3233.64 & 3215.76 & 3213.53 & 3221.97 \\
\hline 3214.44 & 3292.05 & 3227.92 & 3236.02 & 3492.17 & 3242.14 & 3452.16 & 3402.99 & 3241.40 & 3265.69 & 3228.80 & 3230.13 & 3232.60 \\
\hline 3229.18 & 3455.20 & 3393.37 & 3251.85 & 3587.67 & 3395.57 & 3516.86 & 3503.70 & 3526.70 & 3514.14 & 3389.91 & 3542.83 & 3526.32 \\
\hline 3516.26 & 3518.43 & 3460.80 & 3370.08 & 3636.16 & 3660.41 & 3566.15 & 3552.87 & 3690.62 & 3603.40 & 3664.89 & 3693.52 & 3664.20 \\
\hline 3669.63 & 3670.53 & 3649.41 & 3645.48 & 3647.85 & 3667.66 & 3670.66 & 3659.72 & 3716.67 & 3664.82 & 3666.64 & 3715.19 & 3687.55 \\
\hline 3684.76 & 3732.37 & 3672.37 & 3662.34 & 3709.80 & 3738.73 & 3725.05 & 3743.95 & 3724.94 & 3738.92 & 3716.45 & 3730.59 & 3744.93 \\
\hline
\end{tabular}


Table S5: Calculated ${ }^{13} \mathrm{C}$ chemical shifts (ppm relative to tetramethylsilane) for the 26 different neutral tetracycline structures.

\begin{tabular}{|c|c|c|c|c|c|c|c|c|c|c|c|c|c|c|c|c|c|c|}
\hline & $\mathrm{C1}$ & $\mathrm{C2}$ & C3 & $\mathrm{C4}$ & C4a & C5 & C5a & C6 & C6a & C7 & C8 & C9 & C10 & C10a & C11 & C11a & C12 & C12a \\
\hline N1 & 204.5 & 113.1 & 195.1 & 79.3 & 48.0 & 34.6 & 53.8 & 81.2 & 156.9 & 121.9 & 144.3 & 125.9 & 175.6 & 123.6 & 201.7 & 111.0 & 193.2 & 86.4 \\
\hline N2 & 204.4 & 113.1 & 195.7 & 79.4 & 46.5 & 34.4 & 54.3 & 82.2 & 155.4 & 121.7 & 142.9 & 126.1 & 175.3 & 125.1 & 200.2 & 112.1 & 174.9 & 85.1 \\
\hline N3 & 205.1 & 114.4 & 207.0 & 72.6 & 51.7 & 28.7 & 51.5 & 83.5 & 157.0 & 122.7 & 144.7 & 126.4 & 174.8 & 123.0 & 202.9 & 112.8 & 189.7 & 83.9 \\
\hline N4 & 205.3 & 113.3 & 201.4 & 79.3 & 41.9 & 38.4 & 53.5 & 81.4 & 158.0 & 122.5 & 145.4 & 125.9 & 174.7 & 123.3 & 202.1 & 113.2 & 189.9 & 85.0 \\
\hline N5 & 208.5 & 114.2 & 205.9 & 80.1 & 42.4 & 38.7 & 56.1 & 82.2 & 155.7 & 121.3 & 143.9 & 126.3 & 175.6 & 124.9 & 199.5 & 125.8 & 178.7 & 80.5 \\
\hline N6 & 210.0 & 113.7 & 215.9 & 71.3 & 50.0 & 29.6 & 55.4 & 83.8 & 156.4 & 120.4 & 143.0 & 126.7 & 175.7 & 124.4 & 201.4 & 115.0 & 171.7 & 84.2 \\
\hline Z1 & 187.6 & 114.4 & 187.3 & 89.4 & 46.2 & 34.8 & 54.0 & 81.2 & 157.6 & 122.5 & 145.1 & 125.9 & 174.7 & 123.3 & 201.8 & 111.8 & 190.4 & 86.6 \\
\hline $\mathbf{Z 2}$ & 183.6 & 117.3 & 194.0 & 88.5 & 48.4 & 34.0 & 56.4 & 81.4 & 154.5 & 119.6 & 141.3 & 125.8 & 175.1 & 125.5 & 197.0 & 112.2 & 184.0 & 79.3 \\
\hline $\mathbf{Z 3}$ & 203.9 & 117.8 & 184.8 & 85.4 & 47.5 & 35.2 & 56.0 & 81.4 & 153.6 & 119.3 & 139.6 & 125.5 & 174.9 & 125.9 & 195.9 & 101.0 & 177.1 & 32.4 \\
\hline $\mathbf{Z 4}$ & & 116.4 & & 86.5 & & 40.6 & 54.3 & & & & 143.2 & & 174.9 & & & & & .0 \\
\hline $\mathrm{Z5}$ & 189.5 & 116.2 & 190.1 & 89.0 & 46.8 & 34.1 & 56.5 & 81.9 & 155.3 & 121.0 & 143.4 & 126.1 & 175.5 & 125.1 & 199.0 & 121.6 & 179.7 & 81.6 \\
\hline Z6 & 204.5 & 110.1 & 196.6 & 87.5 & 46.7 & 35.2 & 55.9 & 81.5 & 153.7 & 119.2 & 139.4 & 125.4 & 174.9 & 126.0 & 195.8 & 100.8 & 177.6 & 82.1 \\
\hline $\mathbf{Z 7}$ & 190.2 & 118.8 & 187.4 & 80.1 & 52.5 & 29.8 & & 86.3 & 150.9 & 123.8 & 143.7 & 128.6 & 175.0 & 123.3 & & 109.3 & 195.6 & 83.7 \\
\hline $\mathbf{Z 8}$ & 208.4 & 112.8 & 193.4 & 88.7 & 47.2 & 35.3 & 55.8 & 81.3 & 153.6 & 119.3 & 139.9 & 125.6 & 174.9 & 125.8 & 196.1 & 101.8 & 175.7 & 79.7 \\
\hline Z9 & 196.1 & & & 84.0 & 51.7 & 32.4 & 49.4 & 83.4 & 154.9 & & & & 174.8 & 122.8 & & 111.2 & 194.4 & 83.4 \\
\hline Z10 & 205.5 & 118.6 & 180.0 & 85.6 & 46.7 & 41.8 & 56.9 & 80.9 & 153.2 & 118.5 & 139.0 & 125.3 & 174.9 & 126.4 & 194.7 & 100.2 & 178.1 & 79.5 \\
\hline Z11 & 207.4 & 109.0 & 194.1 & 86.9 & 45.4 & 42.4 & 57.1 & 81.0 & 153.4 & 118.4 & 138.7 & 125.2 & 174.9 & 126.5 & 194.5 & 100.6 & 179.0 & 81.2 \\
\hline Z12 & 187.3 & & & 89.6 & 46.4 & 34.5 & 53.4 & & & & & & 171.2 & & & 108.9 & 209.2 & 88.3 \\
\hline Z13 & 214.6 & 112.5 & 199.0 & 81.2 & 54.5 & 29.9 & 51.0 & 89.2 & 146.3 & 118.8 & 138.4 & 127.9 & 175.7 & 125.9 & 188.6 & 103.5 & 185.1 & 89.0 \\
\hline Z14 & 197.5 & 116.4 & 181.7 & 83.7 & 51.5 & 31.8 & 49.5 & 83.5 & 153.8 & 123.8 & 143.1 & 126.9 & 170.9 & 119.3 & 180.9 & 110.3 & 212.4 & 85.2 \\
\hline Z15 & 191.4 & 118.2 & 186.9 & 79.6 & 52.7 & 29.7 & 48.8 & 86.2 & 149.8 & 124.8 & 142.5 & 128.5 & 171.2 & 120.4 & 179.8 & 110.3 & 213.1 & 85.2 \\
\hline Z16 & 211.5 & 108.7 & 202.4 & 81.2 & 53.4 & 29.3 & 49.8 & 89.5 & 146.6 & 115.9 & 138.0 & 128.1 & 176.2 & 127.3 & 187.5 & 103.3 & 188.3 & 91.0 \\
\hline Z17 & 195.5 & 120.1 & 192.4 & 78.7 & 50.4 & 29.1 & 50.3 & 86.9 & 148.5 & 122.7 & 142.1 & 128.8 & 175.7 & 125.1 & 198.3 & 107.9 & 179.1 & 83.0 \\
\hline Z18 & 215.8 & 108.6 & 195.8 & 85.5 & 52.3 & 33.6 & 53.1 & 85.0 & 150.8 & 117.8 & 138.1 & 125.7 & 174.7 & 126.4 & 189.1 & 103.8 & 191.8 & 92.1 \\
\hline Z19 & 224.5 & 111.9 & 193.1 & 86.0 & 53.4 & 33.6 & 53.5 & 84.8 & 150.7 & 118.0 & 138.5 & 126.0 & 174.7 & 125.9 & 189.6 & 105.4 & 188.6 & 92.1 \\
\hline $\mathbf{Z 2 0}$ & 200.4 & 117.3 & 180.7 & 87.5 & 44.6 & 41.7 & 56.6 & 81.9 & 154.6 & 118.1 & 142.0 & 127.4 & 175.9 & 126.1 & 199.5 & 125.7 & 181.0 & 79.5 \\
\hline
\end{tabular}


Table S6: Calculated ${ }^{15} \mathrm{~N}$ and ${ }^{17} \mathrm{O}$ isotropic magnetic shieldings (ppm) for the 26 tetracycline structures.

\begin{tabular}{|c|c|c|c|c|c|c|c|c|c|c|}
\hline & NMe2H & NH2 & Amid-O & 01 & $\mathbf{O 3}$ & 06 & 010 & 011 & 012 & O12a \\
\hline N1 & 209.09 & 155.04 & 47.80 & -171.04 & 122.05 & 259.10 & 199.08 & 0.39 & 144.72 & 257.37 \\
\hline N2 & 208.46 & 155.02 & 48.41 & -159.89 & 118.58 & 257.30 & 202.55 & -124.22 & 181.31 & 264.88 \\
\hline N3 & 221.43 & 157.94 & 49.92 & -123.09 & 123.21 & 240.60 & 197.28 & -2.04 & 161.80 & 274.53 \\
\hline N4 & 225.96 & 157.69 & 45.96 & -158.79 & 120.93 & 257.33 & 198.03 & -0.92 & 167.38 & 263.36 \\
\hline N5 & 224.07 & 156.11 & 44.83 & -82.66 & 99.14 & 251.17 & 201.29 & -138.15 & 196.19 & 241.26 \\
\hline N6 & 225.04 & 156.76 & 50.15 & -85.92 & 97.77 & 233.29 & 201.98 & -134.75 & 185.19 & 251.27 \\
\hline $\mathrm{Z1}$ & 196.14 & 157.15 & -31.65 & -65.37 & -214.13 & 256.13 & 198.05 & 2.98 & 156.15 & 256.01 \\
\hline $\mathbf{Z 2}$ & 198.34 & 153.95 & -49.47 & 157.41 & -275.47 & 258.83 & 203.37 & -95.99 & 46.47 & 240.38 \\
\hline $\mathbf{Z 3}$ & 200.17 & 152.17 & 50.28 & -194.78 & 133.51 & 266.83 & 203.89 & -73.10 & 7.92 & 252.98 \\
\hline $\mathrm{Z4}$ & 203.98 & 161.59 & -51.48 & -257.33 & 55.38 & 232.87 & 198.27 & 0.12 & 165.83 & 255.34 \\
\hline $\mathbf{Z 5}$ & 195.47 & 155.84 & -37.78 & 19.72 & -246.28 & 252.55 & 202.00 & -130.29 & 171.89 & 237.54 \\
\hline $\mathrm{Z6}$ & 202.52 & 157.66 & 171.13 & -154.79 & -62.85 & 267.41 & 203.93 & -70.65 & 5.26 & 255.20 \\
\hline $\mathbf{Z 7}$ & 201.96 & 159.08 & -45.16 & -41.52 & -115.64 & 244.29 & 194.72 & 3.34 & 146.11 & 268.74 \\
\hline Z8 & 203.01 & 158.00 & 41.29 & 99.62 & -166.51 & 265.58 & 203.71 & -76.18 & 5.81 & 247.91 \\
\hline Z9 & 211.45 & 159.32 & -45.75 & -72.26 & -14.70 & 240.00 & 196.13 & 4.50 & 148.36 & 276.13 \\
\hline Z10 & 199.96 & 152.12 & 53.76 & -199.18 & 166.59 & 270.31 & 203.55 & -64.38 & -19.64 & 245.12 \\
\hline Z11 & 201.60 & 158.06 & 175.21 & -164.29 & 10.29 & 270.97 & 203.83 & -61.23 & -24.27 & 247.69 \\
\hline Z12 & 196.14 & 157.63 & -30.45 & -78.78 & -206.70 & 264.93 & 200.44 & 192.03 & -68.72 & 258.95 \\
\hline Z13 & 203.50 & 159.33 & 45.80 & 92.48 & -104.66 & 242.67 & 194.32 & -24.52 & -157.39 & 270.34 \\
\hline Z14 & 211.36 & 159.39 & -44.81 & -75.83 & -9.20 & 248.30 & 199.16 & 193.85 & -89.20 & 277.61 \\
\hline Z15 & 201.51 & 159.05 & -44.50 & -45.84 & -110.88 & 248.62 & 198.52 & 193.76 & -89.99 & 271.38 \\
\hline Z16 & 204.06 & 159.95 & 175.73 & -130.80 & -21.77 & 234.53 & 191.34 & -14.15 & -171.18 & 280.98 \\
\hline Z17 & 202.93 & 159.35 & -50.60 & 3.25 & -160.02 & 243.53 & 198.05 & -124.71 & 159.35 & 255.68 \\
\hline Z18 & 211.06 & 158.49 & 175.36 & -123.63 & 17.49 & 243.99 & 200.62 & -34.61 & -158.57 & 285.58 \\
\hline Z19 & 210.27 & 159.19 & 43.77 & 89.93 & -44.83 & 245.53 & 200.61 & -40.83 & -155.61 & 273.73 \\
\hline $\mathbf{Z 2 0}$ & 200.00 & 159.79 & -67.12 & -135.96 & 18.57 & 233.56 & 200.73 & -131.78 & 185.26 & 235.25 \\
\hline
\end{tabular}

